\title{
The molecular and cellular signatures of the mouse eminentia thalami support its role as a signalling centre in the developing forebrain
}

\author{
Kevin Kofi Adutwum-Ofosu ${ }^{1,2} \cdot$ Dario Magnani $^{1,3} \cdot$ Thomas Theil $^{1}$ • \\ David J. Price ${ }^{1} \cdot$ Vassiliki Fotaki $^{1}$
}

Received: 25 June 2015/Accepted: 30 September 2015/Published online: 12 October 2015

(C) The Author(s) 2015. This article is published with open access at Springerlink.com

\begin{abstract}
The mammalian eminentia thalami (EmT) (or thalamic eminence) is an embryonic forebrain structure of unknown function. Here, we examined the molecular and cellular properties of the mouse EmT. We first studied mRNA expression of signalling molecules and found that the EmT is a structure, rich in expression of secreted factors, with Wnts being the most abundantly detected. We then examined whether EmT tissue could induce cell fate changes when grafted ectopically. For this, we transplanted EmT tissue from a tau-GFP mouse to the ventral telencephalon of a wild type host, a telencephalic region where Wnt signalling is not normally active but which we showed in culture experiments is competent to respond to Wnts. We observed that the EmT was able to induce in adjacent ventral telencephalic cells ectopic expression of Lef1, a transcriptional activator and a target gene of the Wnt/ $\beta$ catenin pathway. These Lef1-positive;GFP-negative cells expressed the telencephalic marker Foxg1 but not Ascl1, which is normally expressed by ventral telencephalic cells. These results suggest that the EmT has the capacity to activate $\mathrm{Wnt} / \beta$-catenin signalling in the ventral telencephalon and to suppress ventral telencephalic gene
\end{abstract}

Electronic supplementary material The online version of this article (doi:10.1007/s00429-015-1127-3) contains supplementary material, which is available to authorized users.

Vassiliki Fotaki

vfotaki@ed.ac.uk

1 The University of Edinburgh, Centre for Integrative Physiology, Hugh Robson Building, George Square, Edinburgh EH8 9XD, UK

2 Department of Anatomy, College of Health Sciences, University of Ghana, Accra, Ghana

3 Present Address: MRC Centre for Regenerative Medicine, University of Edinburgh, Edinburgh EH16 4SB, UK expression. Altogether, our data support a role of the EmT as a signalling centre in the developing mouse forebrain.

Keywords Mouse - Development - Eminentia thalami . Signalling centre $\cdot \mathrm{Wnt} / \beta$-catenin signalling

\section{Introduction}

The specification and organization of the central nervous system (CNS) start at the gastrula stage of embryonic development. Following the induction of its primordium, the neural plate, by the Spemann-Mangold organizer, the CNS is coarsely patterned along its anteroposterior and dorsoventral axes (reviewed by Kiecker and Lumsden 2012). Neural induction and neural patterning in vertebrates are mediated through signalling molecules (secreted proteins) that emanate from signalling centres (Grove et al. 1998; Houart et al. 1998; Martinez 2001; Martinez and Alvarado-Mallart 1989; Shimamura and Rubenstein 1997).

Local signalling centres are transient structures that usually lie at the boundaries of the tissues they pattern, and secrete molecules that diffuse through adjacent tissue to establish gradients that confer positional information on the cells (Grove and Fukuchi-Shimogori 2003; Kiecker and Lumsden 2005; Wolpert 1996, 2011). Also, in a manner similar to the early organizer, these local signalling centres possess the ability to induce ectopic cell fates in host tissues when heterotopically transplanted (Liu and Joyner 2001; Martinez et al. 1991; Raible and Brand 2004; Shimamura and Rubenstein 1997). The recent combination of genetic and molecular methods with morphological approaches led to the identification of three such local signalling centres in the developing forebrain: the anterior neural ridge (ANR), the cortical hem (hem) and the zona 
limitans intrathalamica (ZLI). The ANR is located in the most rostral part of the neural plate and is a source of Fgf molecules. Ablation of the neural ridge leads to loss of anterior forebrain fates in both mouse and zebrafish (Houart et al. 1998, 2002; Shimamura and Rubenstein 1997). The hem is located in the dorsomedial telencephalon and is rich in expression of Wnt and Bmp molecules (Grove et al. 1998; Lee et al. 2000). It is essential for the specification and patterning of the hippocampal primordium. Gli $3^{-/-}$mutant mice (extratoes) with no hem tissue do not develop a hippocampus (Theil et al. 1999; Tole et al. 2000), while Lhx2-null mouse chimeras develop ectopic hems that induce the formation of multiple hippocampal fields (Mangale et al., 2008; Subramanian and Tole 2009). The ZLI is found in the diencephalic primordium separating the prethalamus from the thalamus. It secretes Shh and is crucial for diencephalic specification and growth (Kiecker and Lumsden 2004; Martinez-Ferre and Martinez 2012; Vieira et al. 2005).

The eminentia thalami (EmT) is a forebrain structure that forms, along with the prethalamus, in prosomere 3 (p3) of the prosencephalon (Puelles and Rubenstein 2003). It is flanked medially by the prethalamus and laterally by the choroid plexus of the lateral ventricle. Its rostral part forms the posterior limit of the interventricular foramen of Monroe, and the caudal part becomes attached to the ventral telencephalon (Abbott and Jacobowitz 1999; Trujillo et al. 2005). The role of the EmT in mammals has not been fully determined. It is postulated that the EmT may be a source of a subtype of Cajal-Retzius cells in the developing neocortex (Meyer 2010; Tissir et al. 2009). Based on its location at the interface between the diencephalon and telencephalon and its transient nature, Abbott and Jacobowitz (1999) suggested that it may act as an organizing centre in forebrain patterning. A recent report proposes that the EmT forms part of the forebrain hem system and may play a role in forebrain patterning and development (Roy et al. 2014).

In this study, we aimed to shed light into the putative role of the EmT as a forebrain signalling centre. Using an extensive battery of signalling molecules, we first examined the expression of members of the Wnt, Fgf and Bmp families in the EmT from E11.5 to E17.5, to determine their temporal and spatial patterns of expression. Members of these developmental control gene families have been implicated in patterning other parts of the CNS (Grove et al. 1998; Shimamura and Rubenstein 1997; Liu and Joyner 2001; Martinez 2001; Martinez et al. 1991; Houart et al. 2002). The EmT was found to express several members of these families, with Wnts being the most broadly and intensely expressed signalling molecules. Next, we examined whether the EmT had the capacity to induce ectopically cell fate changes in the ventral telencephalon when heterotopically transplanted. We observed that the EmT, but not neocortical tissue, was able to induce in ventral telencephalic cells ectopic expression of Lef1, a target gene and transcriptional activator of the $\mathrm{Wnt} / \beta$-catenin pathway. These Lef1-positive cells expressed the telencephalic marker Foxg1, but were negative for the ventral telencephalic marker Ascl1. Our data explore the molecular and cellular properties of the EmT and support the notion that this structure may act as a signalling centre in the mouse developing forebrain.

\section{Materials and methods}

\section{Animals}

Animal care was according to institutional guidelines. Mice were mated, and the morning of the vaginal plug was designated as embryonic day (E) 0.5 . Time-mated pregnant mice were culled by cervical dislocation or overdose of Isoflurane (Merial, UK). Embryos used for gene expression analyses were obtained from wild type female mice of CD1 background. The donor eminentia thalami tissue was from tau-GFP transgenic mouse embryos (Pratt et al. 2000). Embryos were dissected out of the uteri in ice-cold phosphate-buffered saline (PBS). For culture experiments, embryos were dissected in ice-cold $1 \times$ Kreb's buffer.

\section{Organotypic slice culture}

Organotypic slice cultures of the E13.5 embryonic mouse forebrain were done as previously described (Magnani et al. 2010). Brains were removed and placed into ice-cold $1 \times$ Kreb's buffer with $10 \mathrm{mM}$ HEPES buffer (Invitrogen), Gentamicin (Sigma) and Penicillin-Streptomycin (Invitrogen). Brain tissue was embedded in molten $4 \%$ Low Melting Point agarose (Seakem) in PBS at $43^{\circ} \mathrm{C}$ with stirring and was solidified on ice. Tissue was sectioned coronally on a vibratome at a thickness of $300 \mu \mathrm{m}$. Slices were collected into chilled $1 \times$ Kreb's buffer with HEPES and antibiotics. Brain slices were cultured in organ tissue dishes containing $1 \mathrm{ml}$ of medium (Neurobasal, Gibco) supplemented with B-27, glutamine, glucose, penicillin, and streptomycin. For transplantations, brain slices were cultured in Millicell-CM $30 \mathrm{~mm}$ low height membrane inserts (Millipore). Eminentia thalami were dissected out from one or both sides of the brain of donor slices, and transplanted to the ventral telencephalon of host slices. Slices were re-incubated for between 24 and $48 \mathrm{~h}$, after which they were fixed in $4 \%$ paraformaldehyde (PFA) in $0.1 \mathrm{M}$ phosphate buffer (PB) overnight at $4^{\circ} \mathrm{C}$ and processed for immunohistochemistry or in situ hybridization. For activation of $\mathrm{Wnt} / \beta$-Catenin signalling in the ventral telencephalon, slices were cultured on polycarbonate culture membranes ( $8 \mu \mathrm{m}$ pore size; Corning Costar) in the 
presence of either $1 \%$ dimethyl sulfoxide (DMSO) or of 5 , 25 or $50 \mu \mathrm{M}$ CHIR99021 (CHIR) (Cambridge BioScience). Slices were cultured for $24 \mathrm{~h}$, fixed as described above, and processed for immunohistochemistry or in situ hybridization as described below.

\section{Histology, immunohistochemistry, in situ hybridization}

Embryos for gene expression analysis were collected from E11.5 to E17.5. At least two embryos were analysed for each age. Embryos were decapitated and the heads were immersion fixed in $4 \% \mathrm{PFA}$ in $0.1 \mathrm{M} \mathrm{PB}$ overnight at $4^{\circ} \mathrm{C}$. Cultured slices were fixed as described above.

Fixed tissues were processed for immunohistochemistry or in situ hybridization following previously described protocols (Fotaki et al. 2006, 2013).

For DAB and fluorescence immunodetection, antigen retrieval was achieved by microwaving sections in $10 \mathrm{mM}$ sodium citrate buffer. Following DAB reaction, some slides were counterstained with $0.5 \%$ cresyl violet acetate (Sigma). For immunofluorescence, species-specific secondary antibodies conjugated to Alexa fluor-488, 568 or 647 dyes (1:200, Invitrogen) were used to detect primary antibodies. When signal amplification was required, sections were incubated with biotinylated secondary antibodies. Antibody reactions were then revealed using streptavidin-biotin complex conjugated to either 488 or 568 dyes (1:200, Invitrogen). Sections were counterstained with DAPI (1:10000, Sigma). Antibodies used in this study were mouse monoclonals for Ascl1 (1:200, BD Biosciences), Lhx1 and Lhx5 (Lim1+2; 1:100, DSHB) and Pax6 (1:200, DSHB); rabbit polyclonals for Calretinin (1:1000, Swant), Lef1 (1:1000, Cell Signalling) and Tbr2 (1:1000, kindly provided by Prof. R. Hevner); a goat polyclonal for GFP (1:500, Abcam) and a chick polyclonal for GFP (1:200, AVES Labs).

For in situ hybridization, $1 \mu \mathrm{g}$ of each linearized purified DNA template was transcribed using T3, T7 or SP6 RNA polymerase. RNA antisense probes were labelled using the digoxygenin RNA labelling mix (Roche) according to the manufacturer's instructions. The following riboprobes were used in this study: Bmp4, Bmp6, Fgf8, Fgf15, Mkp3, Spry1, Wnt3a, Wnt7a, Wnt7b, Wnt8b, Wnt9a, Axin2, Lef1, Sfrp2, Ngn2 and Foxg1.

\section{Quantitation of Lef1-positive and Ascl11-positive cells}

To determine the effect of the different concentrations of CHIR on Lef1 induction and upregulation as well as Ascl1 downregulation, Lef1-positive, Ascl1-positive and Lef1;Ascl1-positive cells were counted in five representative sections of every slide using the Image $\mathrm{J}$ 1.46r software (NIH) and the mean determined (from fifteen sections counted from three slices) for each of the concentrations used. Means of the various concentrations were then compared by a one-way Anova using the Graphpad Instat3. $P$ values less than 0.05 were considered significant.

\section{Results}

\section{Analysis of expression of molecular markers of the EmT}

The EmT is a transient structure of the developing mammalian forebrain, which, in a coronal plane, is flanked dorsomedially by the prethalamus and laterally by the choroid plexus (Fig. 1a and Online Resource 3), while in a sagittal plane the EmT lies caudal to the choroid plexus and rostral to the prethalamus (Fig. 1b). To demarcate the EmT and gain a better insight into how it changes during development, we performed immunofluorescence and in situ hybridization using markers that are expressed in this structure at different developmental stages. At E12.5, double immunofluorescence for Pax6 and Calretinin revealed strong Pax6 staining in the ventricular zone (VZ) of the EmT and the prethalamus and Calretinin staining in the EmT cell fibres (Fig. 1c, d) (Abbott and Jacobowitz 1999; Fotaki et al. 2006; Retaux et al. 1999). Double immunofluorescence with an antibody that recognises both $\operatorname{Lhx} 1$ and Lhx 5 and Calretinin revealed strong expression of Lhx $1 \& 5$ in the layer of differentiated neurons of the EmT that was also positive for Calretinin as well as the VZ of the EmT (Fig. 1e, f). Double immunofluorescence for the transcription factors Tbr2 and Ascl1 revealed Tbr2 expression in the layer of differentiated neurons of the EmT, while Ascl1 expression was restricted to the prethalamus (Fig. 1g). Ngn2 mRNA staining was found at the $\mathrm{VZ}$ of the EmT but was not detected in the adjacent prethalamus (Flames et al. 2007), thus allowing us to distinguish the medial boundary of the EmT (Fig. 1h, i).

At E14.5, the VZ of the EmT marked by strong Pax6 protein and Ngn2 mRNA was significantly thinner than that observed at younger stages (Fig. $1 \mathrm{j}$, k compare $1 \mathrm{~d}$ to $1 \mathrm{j}$ and $1 \mathrm{~h}$ to $1 \mathrm{k}$ ). At E15.5 and E16.5, Pax6 and Ngn2 expression in this zone was each detected as a stripe of staining ventral to the choroid plexus at all rostrocaudal levels (arrows in Fig. 11, $\mathrm{m}$ and not shown).

\section{Expression of Wnt, Fgf and Bmp genes in the EmT}

The hypothesis that the EmT acts as a signalling centre predicts that it expresses one or more signalling molecules. To examine this, we analysed the expression of members of the Wnt, Fgf and Bmp families of signalling molecules. 

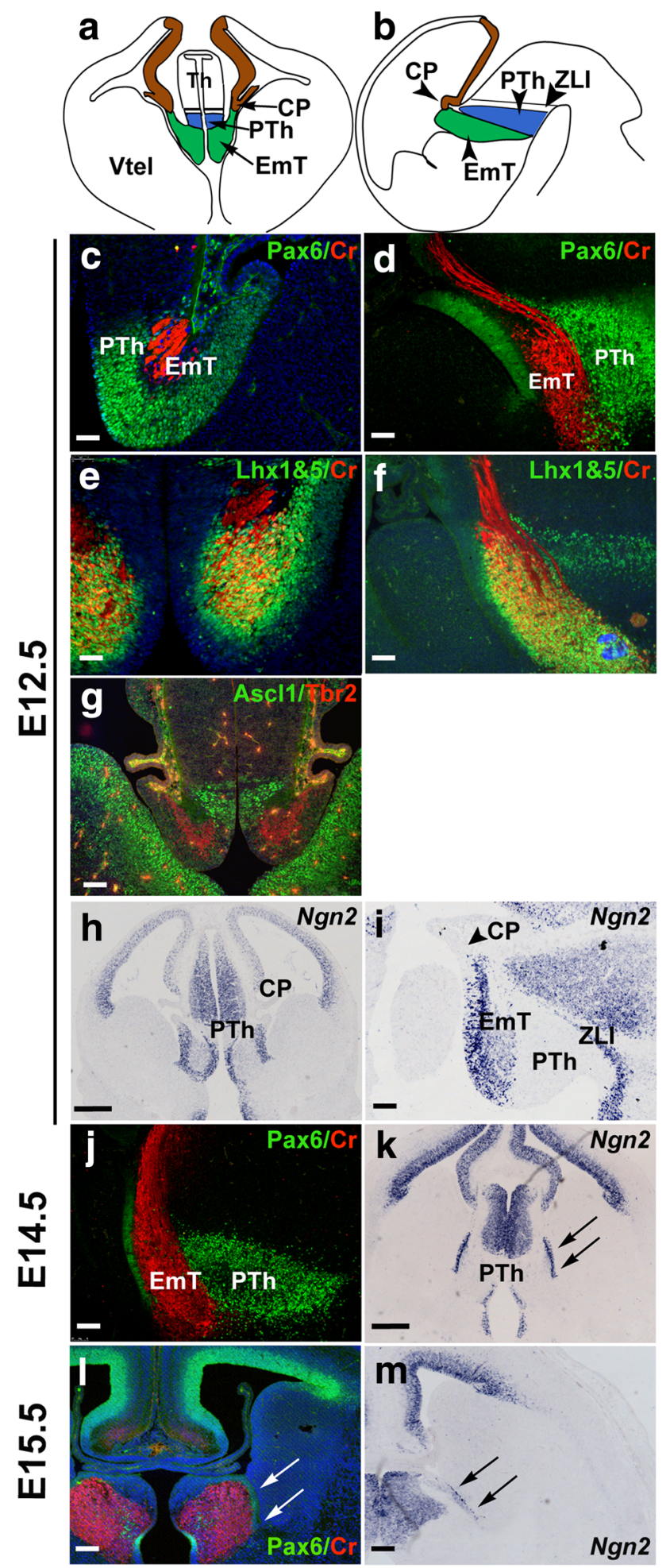

We first examined the expression of members of the Wnt family of signalling molecules. Out of the nineteen members, we examined the expressions of Wnt $3 a, W n t 7 a$, $W n t 7 b$, Wnt8b and Wnt9a which were more likely to be expressed in the EmT based on results from database
4Fig. 1 Expression of molecular markers of the EmT. a, b Schematic representations of a coronal (a) and a sagittal section (b) of an E12.5 forebrain. The prethalamus (blue) is located dorsal (a) and medial (b) to the EmT (green). Brown staining outlines the dorsomedial telencephalon. In $\mathbf{b}$ and all following sagittal sections $(\mathbf{d}, \mathbf{f}, \mathbf{i}, \mathbf{j})$, rostral is to the left and dorsal is to the top. c, $\mathbf{d}$ Pax6 (green) and Calretinin (CR) (red) double immunofluorescence showing expression of these proteins in the EmT at E12.5. In both coronal (c) and sagittal (d) sections, Pax6 is expressed in the proliferative layer while $\mathrm{CR}$ is expressed by the postmitotic cells and fibres of the EmT. Pax6 is also expressed in the prethalamus. e, f Lhx1\&5 (green) and CR (red) immunofluorescence reveals strong Lhx $1 \& 5$ expression in both the proliferative and differentiating layers of the EmT at E12.5 in both coronal (e) and sagittal sections (f). g Ascl1 (green) and Tbr2 (red) immunofluorescence on coronal E12.5 sections reveals Tbr2 expression within the EmT. Ascl1 is found in the adjacent prethalamus. $\mathbf{h}$, i In situ hybridization for Ngn2 at E12.5 in both coronal (h) and sagittal (i) sections. At this stage, Ngn2 mRNA expression distinguishes the EmT from both the prethalamus medially and choroid plexus (arrowhead) laterally. j, I Expression of Pax6 (green) and CR (red) in the EmT in a sagittal section at E14.5 (j) and a coronal section at E15.5 (I). Pax6 and CR still mark the proliferative and postmitotic layers, respectively. k, m Ngn2 expression is still seen in the ventricular zone laterally (arrows) and medially, marking its boundary with the choroid plexus and prethalamus, respectively, both in a coronal section at E14.5 (k) and E15.5 (m). Blue colour in (l) is DAPI used as counterstain. Abbreviations: $C P$ choroid plexus, PTh prethalamus, Th thalamus, Vtel ventral telencephalon, $Z L I$ zona limitans intrathalamica. Scale bars $\mathrm{h}, \mathrm{k}=250 \mu \mathrm{m} ; \mathrm{g}, \mathrm{i}, \mathrm{l}$ and $\mathrm{m}=100 \mu \mathrm{m} ; \mathrm{c}-\mathrm{f}, \mathrm{j}=50 \mu \mathrm{m}$

searches (Genepaint: http://genepaint.org/; The Jackson Laboratory: http://www.jax.org/; Allen Brain atlas: http:// www.brain-map.org/; EMAGE: http://emouseatlas.org/ emage/). In our study, we also included Axin2 and Lef1 which are downstream target genes of the pathway, and Sfrp2, a negative regulator of the pathway (Hsu et al. 1998; Jho et al. 2002; Porfiri et al. 1997). Expression was studied along the rostrocaudal axis from E11.5 until gene expression was not evident. Unless otherwise stated, gene expression for each specific gene was found along the entire axis.

Results of in situ hybridization on E11.5 forebrain tissue showed that at this stage the EmT expresses most of the Wnt and Wnt-related genes analysed. Wnt $7 b$ mRNA expression in the EmT was strong and involved the entire thickness of the neuroepithelium. It extended from its medial extent, where it was continuous with a similar strong expression in the prethalamus, towards its lateral aspects (Fig. 2a). Expression was detected in the cortical hem of the dorsomedial telencephalon, but not in the adjacent choroid plexus of the lateral ventricle (Fig. 2a). Wnt $8 b$ mRNA was detected as a strong homogenous staining involving the neuroepithelium of the EmT, the choroid plexus of the lateral ventricle, the cortical hem and the rest of the medial pallium (Fig. 2e). Expression in the EmT appeared strong throughout, except at its most medial 
extent bordering the prethalamus (Fig. 2e). Weak Wnt9a mRNA was detected in the lateral part of the caudal EmT (Fig. 2i). Wnt3a and Wnt7a were not detected at this stage (not shown). Axin2 mRNA expression in the EmT was strong but was found only in the most lateral aspect of the EmT (Fig. 2m). This strong expression was continuous with a similar expression in the developing choroid plexus and cortical hem in the dorsomedial telencephalon. Sfrp2 expression formed a high-medial to low-lateral gradient, but did not involve the most lateral aspects bordering the choroid plexus (Fig. 2u). Lefl mRNA was not detected in the EmT at this stage, but some mRNA was detected in the prethalamus medial to it (Fig. 2q).

At E12.5, the genes detected at E11.5 were still expressed in the EmT. The pattern and level of expression of most of these genes at this stage were similar to those observed at E11.5 (Fig. 2b, f, j, n, v). However, Wnt8b mRNA expression in the EmT was no longer homogeneous but formed a gradient with the highest levels detected in the lateral EmT, and the lowest level in the medial part bordering the prethalamus (Fig. 2f). Also, there was what appeared to be a very weak Lefl mRNA expression in the caudo-lateral part of the EmT at this stage (Fig. 2r). In addition, weak Wnt $3 a$ expression was detected in the most lateral part of the EmT adjacent to the choroid plexus in the middle and caudal sections (not shown). Wnt7a was also detected at this stage in middle and caudal sections but, unlike Wnt3a, it was detected in the medial half of the EmT (not shown).

At E14.5, most expression patterns were similar to those observed at E12.5 (Fig. 2c, g, k, o, s, w). However, for $W n t 7 b$, Wnt $8 b$ and Wnt $9 a$, the strength of staining appeared lower than that observed at younger stages (Fig. 2c, g, k). The VZ marked by Wnt7b, Wnt8b, Axin2 and Sfrp2 also appeared thinner (Fig. 2c, g, o, w). Wnt3a and Wnt7a were not detected in the EmT at E14.5 (not shown).

Expression of $W n t 7 b$ mRNA was very weak in the EmT at E15.5 (Fig. 2d) and E16.5 (not shown) and was not identifiable at E17.5. Wnt8b, Wnt9a and Lef1 mRNA were not detected in the EmT from E15.5 onwards (Fig. 2h, 1, t). Expression of Axin2 and Sfrp2 mRNAs in the EmT at E15.5 appeared weaker than that observed at E14.5 (Fig. 2p, x) and was not detected beyond E15.5 (not shown).

All the above $W n t$ and $W n t$-related genes were expressed in the VZ. Wnt7b showed an additional weak expression in the mantle layer at E12.5 (Fig. 2b).

We then examined expression of members of the Fgf and Bmp signalling pathways in the EmT (Fig. 3). Based on the results using the same databases as for the study of Wnts, we restricted our analysis to expression of two members of each family: Fgf8 and Fgfl5 for the Fgfs
(Fig. 3a-g) and Bmp4 and Bmp6 for the Bmps (Fig. 3n-s). We also included in our study expression of $M k p 3$ and Spry1, which are downstream targets of Fgf signalling (Mason 2007; Suzuki-Hirano et al. 2010) (Fig. 3h-m).

At E11.5, the intensity of $F g f 8$ mRNA staining was high in the rostral EmT (Fig. 3a), but low in the middle and absent in the caudal EmT (not shown). Weak Fgf15 mRNA expression was detected in the middle and caudal parts of the medial EmT (Fig. 3e), but not in the rostral part. Mkp3 mRNA expression in the EmT was strong at all rostrocaudal levels and appeared to form a high-medial to lowlateral gradient (Fig. 3h). Spryl mRNA was also found in the entire neuroepithelium at all rostrocaudal levels of the EmT, although expression was weak (Fig. 3k). The above molecules were not detected in the neighbouring choroid plexus and cortical hem. Bmp4 and Bmp6 were expressed in the EmT, as well as the cortical hem, choroid plexus and epithalamus. Expression in the EmT was weaker than that detected in the epithalamus, and was confined to its most lateral aspect bordering the choroid plexus (Fig. 3n, q).

At E12.5, the above described $F g f$ and Bmp genes were still expressed in the EmT (Fig. 3b, f, i, 1, o, r). Moreover, the patterns of expression of these genes were similar to those observed at E11.5. At E14.5, Fgf8, Mkp3, Spryl and Bmp4 were still expressed in the EmT (Fig. 3c, j, m, p). However, the intensity of staining seen at this stage appeared weaker and the VZ marked by these genes was also thinner than seen at younger ages. Fgf15 and Bmp6 were not detected in the EmT at this stage (Fig. 3g, s). At E15.5, only Fgf8 was still detected in the EmT (Fig. 3d).

To summarize, all the above members of the Wnt/ $\beta$ catenin signalling pathway were found in the $\mathrm{VZ}$ of the EmT, where staining decreased as development progressed and ceased for most of them by E15.5. Similar results were obtained for the molecules of the Fgf and Bmp signalling pathways, except that most of them were not detected beyond E14.5. The above results are summarized in Table 1.

\section{Effects of small molecule activation of Wnt/ $\boldsymbol{\beta}$-catenin signalling in the ventral telencephalon}

Analyses of gene expression in the EmT showed some distinct and some overlapping expression domains of Wnt, Fgf and Bmp genes. The Wnt genes showed stronger expression during EmT development compared to those of the Fgf and Bmp families. For this reason, we chose to follow up on the role of $\mathrm{Wnt} / \beta$-catenin signalling pathway in EmT function.

To examine whether the Wnt molecules expressed in the EmT have a signalling capacity, we transplanted EmT tissue into the ventral telencephalon to investigate if $\mathrm{Wnt}$ signals from the EmT were able to induce any cell fate 


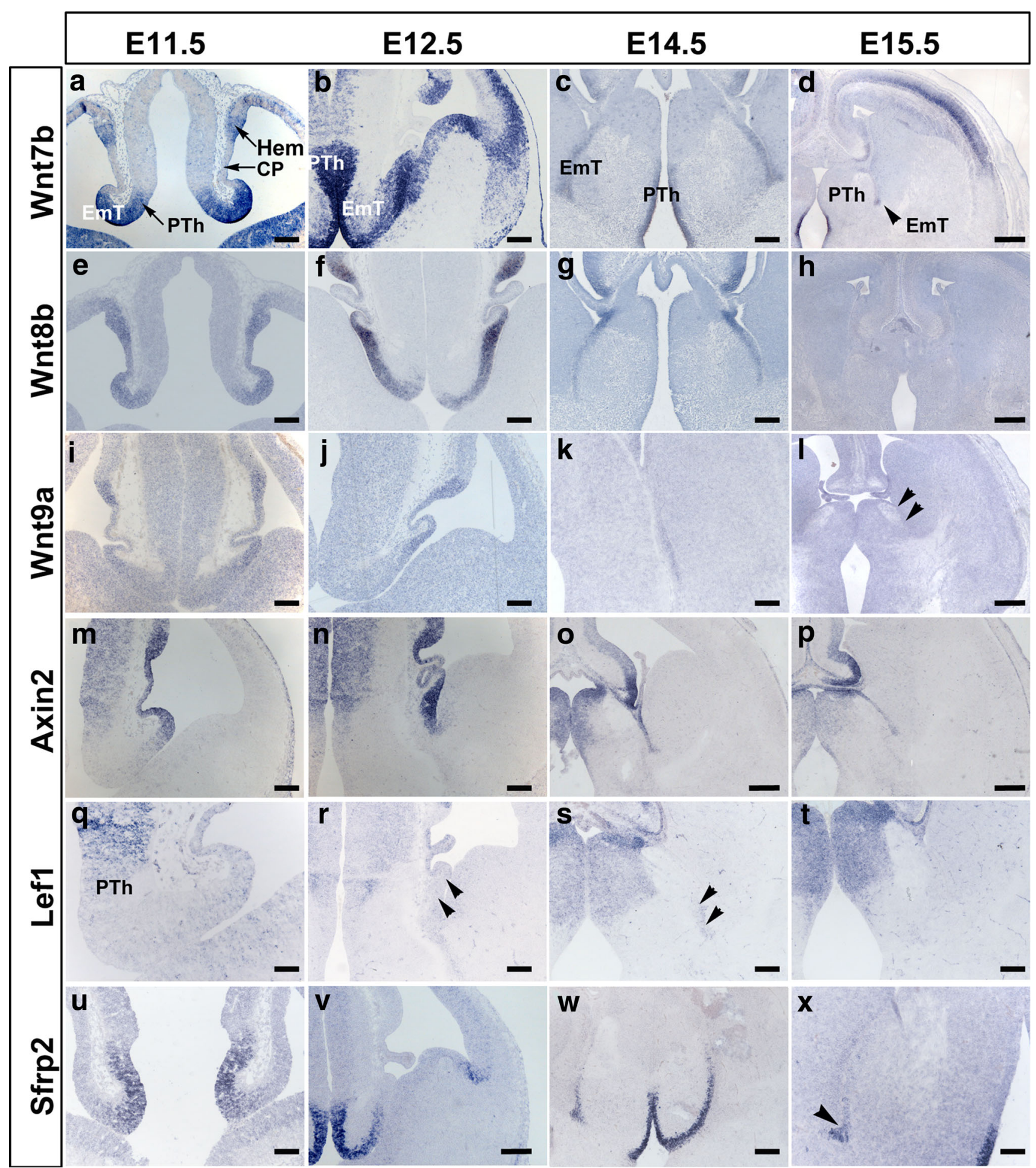

changes in this region (see following section). We chose the ventral telencephalon as it normally does not express significant levels of $\mathrm{Wnt} / \beta$-catenin target genes (Backman et al. 2005; Fotaki et al. 2011; Maretto et al. 2003).

Our first step was to test whether the ventral telencephalon was competent to respond to $\mathrm{Wnt} / \beta$-catenin signalling. For this, we activated the pathway in telencephalic slices by treating them in culture with three different concentrations of CHIR 99021 (CHIR) (5, 25 and $50 \mu \mathrm{M}$ ) in $1 \%$ Dimethyl sulfoxide (DMSO) to dose-dependently inhibit GSK3 $\beta$ activity. CHIR is a substituted dihydropyromidine whose ability to inhibit GSK3 $\beta$ 
4Fig. 2 Expression of Wnt and Wnt-related genes in the EmT from E11.5. a-d $W n t 7 b$ expression. Strong $W n t 7 b$ mRNA is detected in the EmT at E11.5 (a) and 12.5 (b) and is downregulated at E14.5 (c) and E15.5 (arrowhead in d). e-h Wnt $8 b$ expression. Strong Wnt $8 b$ mRNA is detected from 11.5 to $14.5(\mathbf{e}-\mathbf{g})$, after which it is no longer detectable in the EmT (h). i-l Wnt9a expression. Weak Wnt $9 a$ mRNA is detected in the caudo-lateral EmT from E11.5 to E14.5 (i-k), after which it is no longer detected (arrowheads in $\mathbf{l}$ mark the lateral limit of the EmT at E15.5). m-p Axin2 expression. Axin2 mRNA expression is seen in the lateral aspect of the EmT from E11.5 to E15.5. q-t Lef1 expression. At E11.5 (q), Lef1 mRNA is not detected in the EmT, but it is found in the prethalamus medial to it. From E12.5 to E14.5 (r, s), very weak Lef1 mRNA is detected in the lateral aspect of the EmT (arrowheads). This weak expression is not detected from E15.5 onwards (t). $\mathbf{u}-\mathbf{x}$ Sfrp 2 expression. Strong Sfrp 2 mRNA is detected in the EmT from E11.5 to E14.5 (u-w), and becomes weak at E15.5 (arrowhead in $\mathbf{x}$ ). Abbreviations: $C P$ choroid plexus, PTh prethalamus, Th thalamus, Vtel ventral telencephalon. Scale bars $\mathrm{d}, \mathrm{h}$, $1, \quad \mathrm{p}=200 \mu \mathrm{m} ; \mathrm{a}-\mathrm{c}, \mathrm{e}-\mathrm{g}, \mathrm{i}-\mathrm{k}, \mathrm{m}, \mathrm{n}, \mathrm{r}-\mathrm{t}, \mathrm{v}, \mathrm{w}=100 \mu \mathrm{m} ; \mathrm{u}$, $\mathrm{x}=50 \mu \mathrm{m} ; \mathrm{q}=25 \mu \mathrm{m}$

facilitates the cytoplasmic accumulation and subsequent translocation to the nucleus of $\beta$-catenin, which is required for the transcription of downstream targets of the Wnt/ $\beta$ catenin pathway (Ring et al. 2003). Our controls were slices that were treated in culture with $1 \%$ DMSO.

Lef1 is a transcriptional activator in the $\mathrm{Wnt} / \beta$-catenin signalling pathway (Hsu et al. 1998; Porfiri et al. 1997) and is expressed in the thalamus and the dorsal and dorsomedial telencephalon (Fig. 4a, $\mathrm{a}^{1}$ ) (Fotaki et al. 2011; Galceran et al. 2000). DAB immunohistochemistry with an antibody for Lef1 revealed a small number of Lef1-positive cells in the VZ of the lateral ganglionic eminence (LGE), located at a distance from the pallial-subpallial boundary (PSPB) (Fig. 4a, $\mathrm{a}^{1}$ ). These cells express very low levels of Lef1 compared to the Lef1 heavily stained cells in the dorsomedial telencephalon (Fig. $4 \mathrm{a}^{1}$ ) and were not detectable using Lef1-immunofluorescence (not shown). To determine the level of activation of $\mathrm{Wnt} / \beta$-catenin signalling in the ventral telencephalon, we analysed in both control and experimental slices Lef1 expression in this region.

In DMSO-treated slices, Lef1 expression was detected in the VZ of the LGE (Fig. $4 b, b^{1}$ ) at a similar position to that described above (Fig. 4a, $\mathrm{a}^{1}$ ). However, expression was stronger compared to non-treated sections (compare Fig. $4 b^{1}$ to $4 a^{1}$ ), but it was still lower than Lef1 expression in the dorsomedial telencephalon from the same section (Fig. 4b, $\left.b^{1}\right)$. A mean of $245.13( \pm \mathrm{SD}=38.02)$ Lef1positive cells per section was counted in the ventral telencephalon of these control slices (Table 2).

In both $5 \mu \mathrm{M}$ and $25 \mu \mathrm{M}$ CHIR-treated slices, strong Lef1 expression was detected in the VZ and the superficial mantle layer of the ventral telencephalon (Fig. 4c, d). The intensity of staining seen in the ventral telencephalon at these two concentrations was similar to each other and appeared higher than that seen in DMSO-treated slices (compare Figs. $4 c^{1}$ and $d^{1}$ to $4 b^{1}$ ). Cell counts revealed a statistically significant increase of $39 \%(P<0.001)$ and $60 \%(P<0.001)$ in Lef1-positive cells in slices treated with 5 and $25 \mu \mathrm{m}$ CHIR, respectively, compared to the control group (Table 2). In $50 \mu \mathrm{M}$ CHIR-treated slices, very strong Lef1 expression was detected in the VZ as well as in the mantle layer of the ventral telencephalon (Fig. 4e, $\left.\mathrm{e}^{1}\right)$. Moreover, Lef1 expression in the VZ involved its entire mediolateral extent, and included the PSPB (Fig. $4 \mathrm{e}^{1}$ ). Numbers of Lef1-positive cells in the ventral telencephalon were $\sim 192 \%$ greater than those found in the control $(P<0.001)$ (Table 2$)$. These results confirm that the ventral telencephalon is competent to respond to $\mathrm{Wnt} / \beta$-catenin signalling.

We then examined the expression of Foxg1 and Ascl1 to study how the increase in Lef1 staining in the ventral telencephalon may affect distribution of these proteins in this region. In wild types after E12.5, Foxg1 is detected at high levels in both the dorsal and ventral telencephalon (Fotaki et al. 2006; Hanashima et al. 2002; Tao and Lai 1992; Xuan et al. 1995), while Ascl1 is expressed at high levels in the ventral telencephalon (Guillemot and Joyner 1993; Lo et al. 1991; Porteus et al. 1994).

In both DMSO and CHIR-treated slices, Foxgl was expressed in the entire ventral telencephalon forming a gradient, with expression in the VZ being higher than expression in the mantle layer (not shown). Also, the intensity of Foxgl staining seen in sections of all CHIRtreated slices was similar to that seen in DMSO-treated slices (Fig. 4f-h). Lef1 expression was as described earlier for each of the concentrations used. In both DMSO and CHIR-treated slices, Lef1 was detected in the nuclei of some cells in the $\mathrm{VZ}$ and the mantle layer of the ventral telencephalon. In these cells, the Lef1-positive nuclei (brown stain in Fig. 4f-h) were surrounded by cytoplasmic Foxg1 mRNA (purple stain in Fig. 4f-h).

In both control and experimental groups, Ascl1-positive cells were detected in the VZ and mantle layer of the ventral telencephalon at all rostrocaudal levels (Fig. 4i-1). Ascl1 expression extended medially from the subpallial side of the PSPB and included the entire mediolateral extent in both zones. However, the number of cells expressing Ascl1 differed from one group to the other (Table 3). A mean of $215( \pm \mathrm{SD}=44.71)$ Ascl1-positive cells per section was counted in the ventral telencephalon of DMSO-treated slices. In the VZ, a few of these Ascl1positive cells were also Lef1-positive, but the majority were single-labelled (Fig. $4 i^{2}$ ). Ascl1-positive cells in the mantle layer were all single-labelled. In $5 \mu \mathrm{M}$ CHIRtreated slices, Ascll expressing cells present in the ventral telencephalon were $\sim 3 \%$ fewer than those counted in DMSO-treated slices and this difference was not statistically significant (Table 3). Many of the Ascl1-expressing cells detected were single-labelled (Fig. $4 \mathrm{j}^{2}$ ). In 25 and 


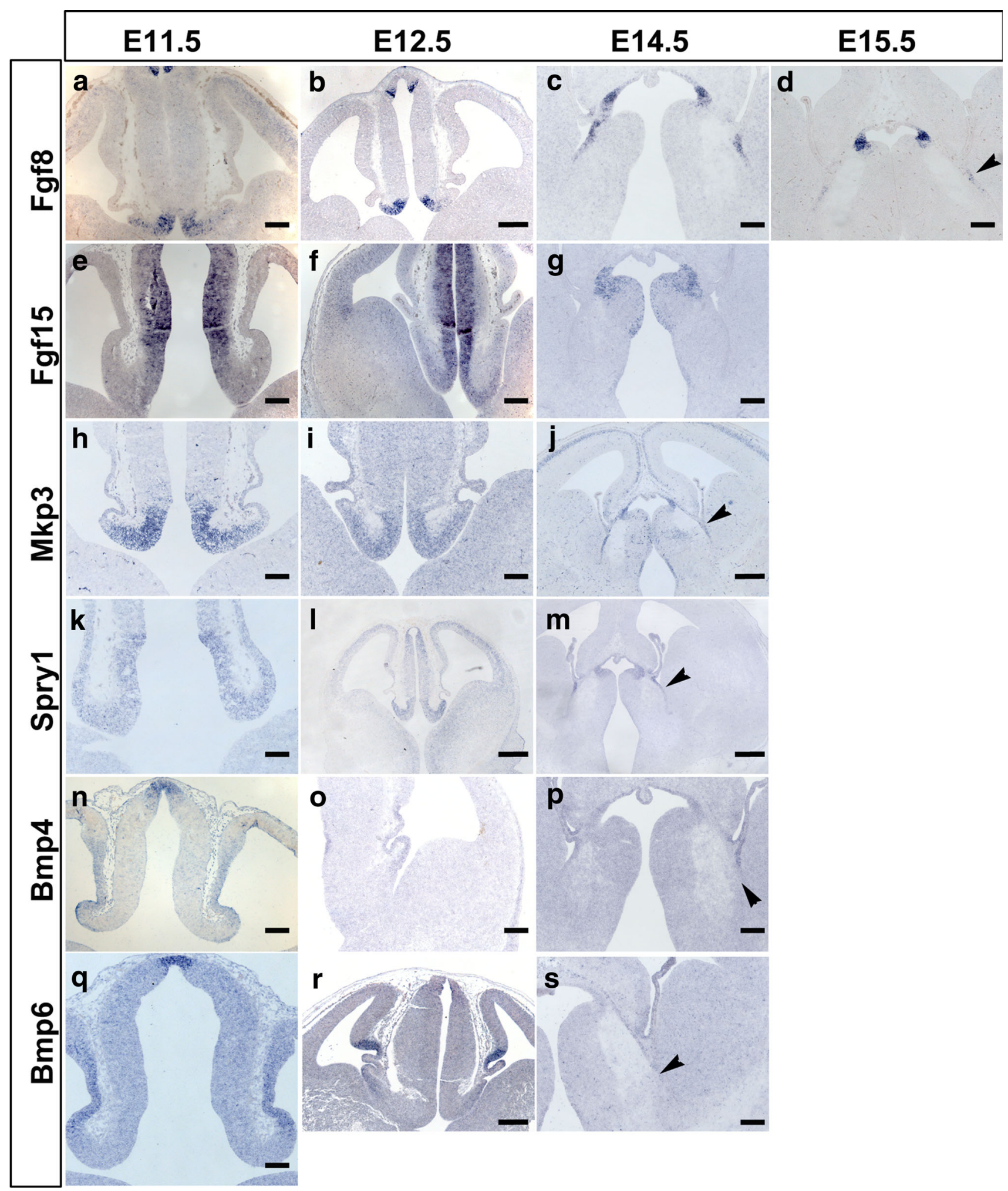

Fig. 3 Expression of Fgf and Bmp-related genes in the EmT from E11.5 to E15.5 a-d $F g f 8$ expression. Strong $F g f 8$ mRNA expression detected in the EmT at E11.5 (a) and 12.5 (b), is downregulated at E14.5 (c) and E15.5 (d). e-g Fgf15 expression. Fgf15 mRNA is detected in the middle and caudal EmT at E11.5 (e) and E12.5 (f) but is no longer detectable in the EmT at E14.5 and beyond (g). hj Strong $M k p 3$ mRNA is expressed in the entire rostrocaudal extent of the EmT till E14.5. Sections shown are from the middle portion of the EmT. k-m Spryl mRNA is expressed in the EmT from E11.5 to E14.5. n-p Bmp4 mRNA expression is detected in the most lateral tip of the EmT till E14.5. q-s Bmp6 mRNA is expressed in the lateral EmT at E11.5 and E12.5 but not at E14.5 (arrowhead in s). Scale bars $\mathrm{b}, \mathrm{j}, \mathrm{l}, \mathrm{m}, \mathrm{r}=200 \mu \mathrm{m} ; \mathrm{a}, \mathrm{c}-\mathrm{i}, \mathrm{k}, \mathrm{n}-\mathrm{p}, \mathrm{q}, \mathrm{s}=100 \mu \mathrm{m}$ 
Table 1 Summary of gene expression in the EmT along the rostrocaudal axis

\begin{tabular}{llllllllllllll}
\hline & & Bmp4 & Bmp6 & Fgf8 & Fgf15 & Mkp3 & Spry1 & Wnt7b & Wnt8b & Wnt9a & Axin2 & Lef1 & Sfrp2 \\
\hline E11.5 & $R$ & + & + & ++ & n.d & ++ & + & +++ & +++ & n.d & ++ & n.d & ++ \\
& $M$ & + & + & + & + & ++ & + & +++ & +++ & n.d & ++ & n.d & ++ \\
& $C$ & + & + & n.d & + & ++ & + & +++ & +++ & + & ++ & n.d & ++ \\
E12.5 & $R$ & + & + & ++ & n.d & ++ & ++ & +++ & +++ & n.d & ++ & n.d & +++ \\
& $M$ & + & + & + & + & ++ & ++ & +++ & +++ & n.d & ++ & n.d & +++ \\
& $C$ & + & + & n.d & + & ++ & ++ & +++ & +++ & + & ++ & + & +++ \\
E14.5 & $R$ & + & n.d & + & n.d & + & + & ++ & ++ & n.d & ++ & n.d & ++ \\
& $M$ & + & n.d & + & n.d & + & + & ++ & ++ & n.d & ++ & n.d & ++ \\
& $C$ & + & n.d & n.d & n.d & + & + & ++ & ++ & + & ++ & + & ++ \\
E15.5 & $R$ & n.d & n.d & + & n.d & n.d & n.d & + & n.d & n.d & + & n.d & + \\
& $M$ & n.d & n.d & + & n.d & n.d & n.d & + & n.d & n.d & + & n.d & + \\
& $C$ & n.d & n.d & n.d & n.d & n.d & n.d & + & n.d & n.d & + & n.d & + \\
\hline
\end{tabular}

Legend: $R$ rostral, $M$ middle, $C$ caudal, $n . d$. not detected, + weak, ++ strong, +++ very strong

$50 \mu \mathrm{M}$ CHIR-treated slices, Ascl1-positive cells were significantly reduced by $\sim 46 \%(P<0.001)$ and $62 \%$ $(P<0.001)$, respectively, compared to the number found in the control group (Table 3). The number of Lef1;Ascl1 double-labelled cells was not different between control and DMSO-treated slices (Table 4).

These results confirm that the ventral telencephalon is competent to respond to $\mathrm{Wnt} / \beta$-catenin signalling as shown by the increased expression of Lef1. In addition, this Lef1 upregulation leads to a decrease in the number of Ascl1positive cells that are normally expressed in the ventral telencephalon.

\section{The EmT induces cell fate changes in the ventral telencephalon}

To test whether signals from the EmT are capable of inducing cell fate changes similar to those observed when $\mathrm{Wnt} / \beta$-catenin signalling is upregulated in the ventral telencephalon, EmT tissue from tau-GFP transgenic mice (donor) that ubiquitously express GFP (Pratt et al. 2000) and, therefore, allowed a distinction between donor and host tissue was transplanted into the ventral telencephalon of wild type CD1 mice (host) in culture. The hosts were then analysed for expression of Lef1, Foxg1 and Ascl1 in the ventral telencephalon.

Two groups of EmT transplants were used. One group consisted of 10 slices, each hosting one piece of EmT explant in the ventral telencephalon (Fig. 5d, Group 1). The other group was made up of 10 slices with two EmT explants each (Fig. 6a, Group 2). To control for the specificity of the effects of the EmT tissue in the ventral telencephalon, an additional group of 10 slices each hosting two pieces of cortical explants from the middle third of the dorsolateral telencephalon, where Lef1 expression is very weak, was included in the study (Fig. 5a, Control group). In all three groups, the explants were placed in one hemisphere, and the opposite hemisphere was used as control. To confirm the location of the explants in the hosts, the slices were examined for GFP expression (green staining in Figs. 5, 6). Additionally in cultures hosting EmT explants, Calretinin immunofluorescence was done to confirm that they were indeed EmT tissue (Fig. $6 b, b^{1}$ and not shown).

Double immunofluorescence for GFP (green) and Lef1 (red) revealed that in the control group, Lef1 was not detected in the ventral telencephalon of $9 / 10$ slices (Fig. 5b, $b^{1}, b^{2}$ ). In $1 / 10$ of slices analysed (Fig. 5c), Lef1positive cells were seen in parts of the VZ, though no positive cells were detected in the mantle layer.

Similarly, in cultured slices hosting one EmT explant, Lef1 was not detected in the ventral telencephalon of $9 / 10$ of the slices (Fig. 5e, $\mathrm{e}^{1}, \mathrm{e}^{2}$ ). In only 1/10 of slices analysed, the Lef1-immunoreactive cells were present in the VZ but not in the mantle layer, as indicated by arrowheads in Fig. 5f.

It should be noted that in some cases Lef1 expression was also observed in the VZ of the ventral telencephalon of the control hemisphere with no explant present (arrowhead in Fig. 6e). This is in line with Lef1 expression detected in a group of slices cultured without explant. In 4/20 of these control slices, Lef1 was detected in the VZ of the ventral telencephalon, either on one or both halves of the brain, but it was never observed in the mantle layer (not shown).

In cultured slices hosting two EmT explants, Lef1 staining was detected in the ventral telencephalon in $9 / 10$ of slices analysed. In 2 of these 9 slices, the Lef1-positive cells were only found at the VZ and were not observed in 


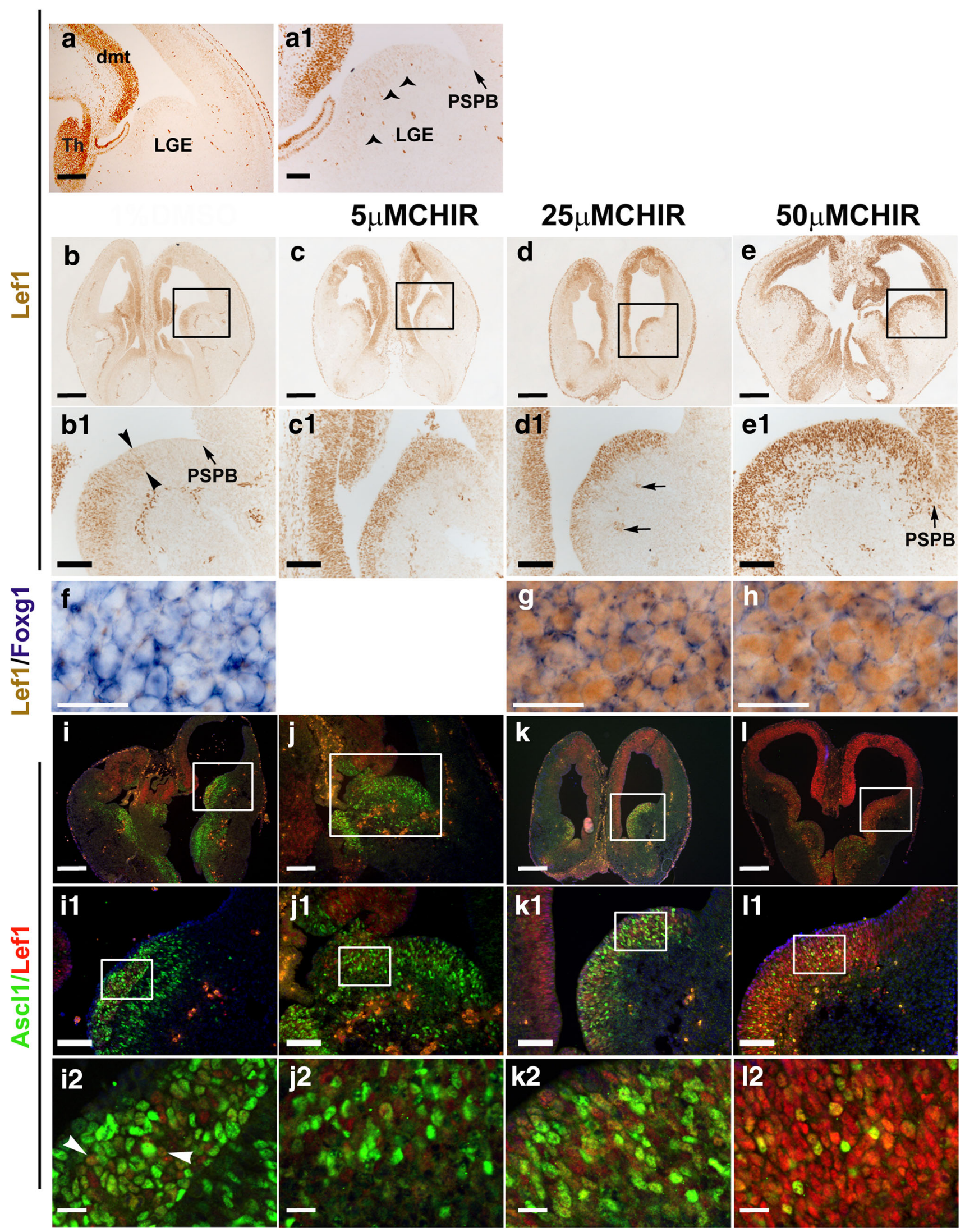


4Fig. 4 Effect of $\mathrm{Wnt} / \beta$-catenin signalling upregulation on gene expression in the ventral telencephalon. $\mathbf{a}, \mathbf{a}^{\mathbf{1}}$ Lef 1 expression in the ventral telencephalon of CD1 mice at E 13.5. Lef1 is strongly expressed in the dmt and Th. Arrowheads in $\mathrm{a}^{1}$ point to faintly expressing Lef1-positive cells detected in the VZ of the ventral telencephalic LGE and at a distance from the PSPB (indicated by an arrow). $\mathbf{a}^{1}$ is a magnification of $\mathbf{a}$. $\mathbf{b}-\mathbf{e}$ Lef1 expression in the ventral telencephalon of treated slices. $\mathbf{b}, \mathbf{b}^{\mathbf{1}}$ Lef1 expression in DMSOtreated slices. Lef1 is detected in parts of the VZ and superficial mantle layer. Staining is not seen at the PSPB but at some distance away from it, as shown with arrowheads in $\mathrm{b}^{1}$. c, $\mathbf{c}^{\mathbf{1}}$ Lef1 expression in slices treated with $5 \mu \mathrm{M}$ CHIR and (d, $\left.\mathbf{d}^{\mathbf{1}}\right) 25 \mu \mathrm{M}$ CHIR. A fairly strong Lef1 expression is seen in the ventral telencephalon. Lef1 expression marks almost the entire VZ and superficial mantle layer beginning from the subpallial side of the PSPB. The arrows in $\mathrm{d}^{1}$ point to blood vessels found in the mantle. $\mathbf{e}-\mathbf{e}^{\mathbf{1}}$ Lef1 expression in $50 \mu \mathrm{m}$ CHIR-treated slices. Very strong Lef1 expression is detected in the ventral telencephalon. Several Lef1-positive cells are seen in the VZ and mantle layer of the ventral telencephalon. In the VZ, Lef1 is also expressed in the PSPB (arrowhead in $\mathrm{e}^{1}$ ). $\mathrm{b}^{1}, \mathrm{c}^{1}, \mathrm{~d}^{1}$ and $\mathrm{e}^{1}$ are magnifications of the regions indicated in $b, c, d$ and e, respectively. $\mathbf{f}-\mathbf{h}$ Lef1 and Foxg1 expression in treated slices. In both control (f) and CHIR-treated groups (g, h) Foxg1-positive cells (blue-in situ hybridization staining) in the ventral telencephalon also express Lef1 (brown-DAB staining). i-l Lef1 and Ascl1 expression in the ventral telencephalon of treated slices. $\mathbf{i}, \mathbf{i}^{\mathbf{1}}$ and $\mathbf{i}^{\mathbf{2}}$ In DMSO-treated slices, Ascll is detected in the VZ and mantle layer of the ventral telencephalon. A few Lef1-positive cells are detected within these zones $\left(\mathbf{i}, \mathbf{i}^{1}\right)$. Most of the Lef1-positive cells are Ascl1-negative and only a few are double-labelled (arrowheads in $\mathrm{i}^{2}$ ). $\mathbf{j}, \mathbf{j}^{\mathbf{1}}$ and $\mathbf{j}^{\mathbf{2}}$ Lef1 and Ascl1 expression in $5 \mu \mathrm{M}$ CHIR-treated slides. Several Ascl1expressing cells are detected in the VZ and mantle. The intensity of staining seen in many of the cells is similar to that seen in i. Many of the cells are single-labelled $\left(\mathbf{j}^{2}\right) . \mathbf{k}, \mathbf{k}^{\mathbf{1}}$ and $\mathbf{k}^{\mathbf{2}}$ Lef1 and Ascl1 expression in $25 \mu \mathrm{M}$ CHIR-treated slides. Fewer Ascl1-expressing cells than those detected in $\mathrm{i}$ and $\mathrm{j}$ are seen in the $\mathrm{VZ}$ and mantle layer. $\mathbf{l}, \mathbf{l}^{\mathbf{1}}$ and $\mathbf{l}^{\mathbf{2}}$ Lef1 and Ascll expression in $50 \mu \mathrm{M}$ CHIR-treated slides. Very few Ascl1-positive cells are seen in the VZ and mantle layer of the ventral telencephalon. On the other hand, many Lef1expressing cells are detected in these zones, many of which are Ascl1negative. Abbreviations: $d m t$ dorsomedial telencephalon, $L G E$ lateral ganglionic eminence, $P S P B$ Pallial-Subpallial Boundary, Th Thalamus, VZ Ventricular zone. Scale bars $\mathrm{b}-\mathrm{e}, \mathrm{k}-\mathrm{n}=250 \mu \mathrm{m} ; \mathrm{a}, \mathrm{b}^{1}-\mathrm{e}^{1}$, $\mathrm{i}^{1}-\mathrm{l}^{1}=50 \mu \mathrm{m}, \mathrm{a}^{1}, \mathrm{f}-\mathrm{h}, \mathrm{i}^{2}-\mathrm{l}^{2}=20 \mu \mathrm{m}$

the mantle layer (not shown). In 7 of the 9 slices, the Lef1positive cells were present in the VZ, as well as the mantle layer (Fig. 6c, $c^{1}, c^{2}, e, e^{1}$ ). Lef1-positive cells were found

Table 2 Lef1-positive cells in the ventral telencephalon of treated slices

Lef1-positive cells per section

\begin{tabular}{|c|c|c|c|c|}
\hline & Mean & STDEV & $\%$ increase & $P$ value \\
\hline DMSO & 245.13 & 38.02 & N/A & N/A \\
\hline $5 \mu \mathrm{M}$ CHIR & 340.80 & 45.56 & 39.03 & $<0.001$ \\
\hline $25 \mu \mathrm{M}$ CHIR & 391.33 & 37.87 & 59.64 & $<0.001$ \\
\hline $50 \mu \mathrm{M}$ CHIR & 715.80 & 102.18 & 192.00 & $<0.001$ \\
\hline
\end{tabular}

Shown are the mean number of cells per section with the standard deviation (STDEV), the \% increase in number of Lef1-positive cells compared to the control (DMSO) group and the $P$ values of each group compared to the control
Table 3 Ascl1-positive $(+)$ cells in the ventral telencephalon of treated slices

Ascl1-positive cells per section

\begin{tabular}{lcccl}
\hline & Mean & STDEV & $\%$ Ascl1-+ & $P$ value \\
\hline DMSO & 215.40 & 44.71 & 100.00 & N/A \\
$5 \mu \mathrm{M} \mathrm{CHIR}$ & 209.60 & 28.76 & 97.31 & $>0.05$ \\
$25 \mu \mathrm{M}$ CHIR & 115.60 & 12.35 & 53.67 & $<0.001$ \\
$50 \mu \mathrm{M}$ CHIR & 82.27 & 11.63 & 38.19 & $<0.001$
\end{tabular}

Shown are the mean number of cells per section with the standard deviation (STDEV), the \% number of Ascl1-positive cells and the $P$ values of each group compared to the control (DMSO) group

Table 4 Lef1 and Ascl1 (Lef1;Ascl1) double-positive cells in the ventral telencephalon of treated slices

Lef1;Ascl1-positive cells per section

\begin{tabular}{llll}
\hline & Mean & STDEV & $P$ value \\
\hline DMSO & 12.33 & 3.31 & N/A \\
$5 \mu \mathrm{M}$ CHIR & 13.83 & 4.37 & $>0.05$ \\
$25 \mu \mathrm{M}$ CHIR & 14.08 & 4.08 & $>0.05$ \\
$50 \mu \mathrm{M}$ CHIR & 14.25 & 5.10 & $>0.05$
\end{tabular}

Shown are the mean number of cells per section with the standard deviation (STDEV) and the $P$ values of each group. No significant differences are observed

in sections where the explants were present, as revealed by their GFP immunoreactivity (compare position of explant in Fig. $6 \mathrm{~b}, \mathrm{c}$ ), and were located around the explants (Fig. 6c, $c^{1}, c^{2}$ ). These Lef1-positive cells were GFP-negative (Lef1 brown staining in Fig. $6 c^{1}$ and $c^{2}$ is found outside the explant, the GFP-positive area shown in Fig. $6 b^{1}$ ). Lef1-positive cells were also found in the VZ and the mantle layer of the ventral telencephalon of sections where no more tissue from the explant could be detected with GFP immunostaining and as before, these Lef1-positive cells were GFP-negative (Fig. 6e, $\mathrm{e}^{1}$ ). The fact that Lef1-expressing cells present in the mantle layer of the ventral telencephalon were GFP-negative indicates that these cells are unlikely to be donor cells and may be ventral telencephalic cells induced to express Lef1.

To gain further insight into the identity of these Lef1expressing cells, we examined whether they expressed the ventral telencephalic marker Ascl1. In sections containing EmT explants, Ascl1 staining was not seen in the explants but was found in cells surrounding them, as shown in Fig. 7c and illustrated in Fig. 7a. Normal expression of Ascl1 and Lef1 was observed in the cultured half of the brain with no explant (not shown). In sections with explants containing Lef1-induced cells, the Lef1-positive cells mingled with the Ascl1-positive cells (Fig. 7d, $d^{1}$ and illustrated in Fig. 7a). Figure $7 d$ and $d^{1}$ depicts sections of 


\section{CULTURED SLICES WITH CORTICAL EXPLANTS (CONTROL GROUP)}
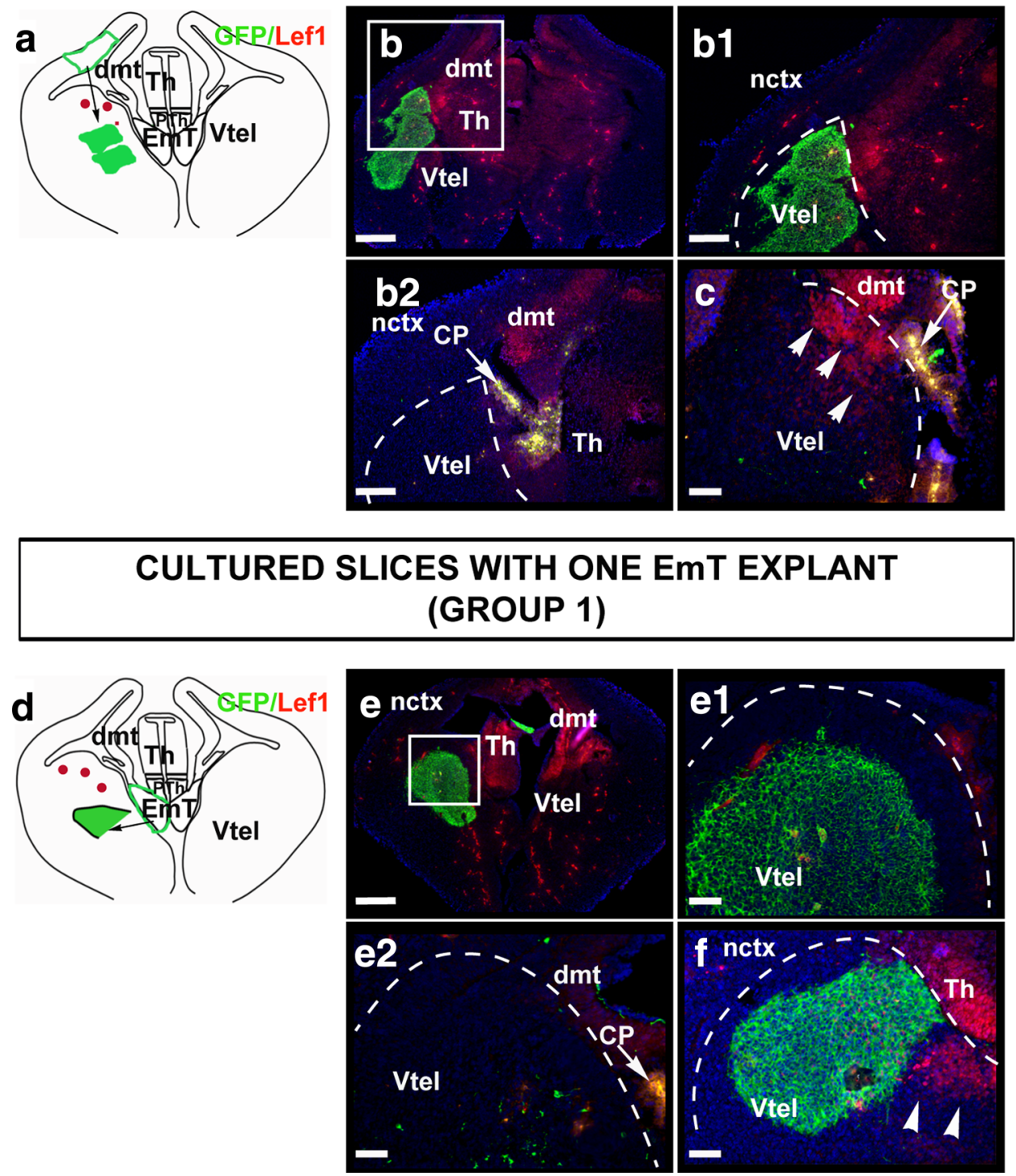

Fig. 5 Lef1 expression in the ventral telencephalon of cultured slices hosting two cortical explants $(\mathbf{a}-\mathbf{c})$ and one EmT explant $(\mathbf{d}-\mathbf{f})$. a A schematic representation of Lef1 expression in slices hosting two cortical explants. The region of the tau-GFP donor tissue from which cortical explants were taken from is shown in green. In 9/10 of slices analysed, Lef1 staining was not seen in the ventral telencephalon. In the remaining 1/10 slices, Lef1 staining was seen in the ventricular zone but not in the mantle layer. b, c Sections of two slices from this group with two tau-GFP-positive cortical explants each on the left hemisphere. $b^{1}$ is a higher magnification of $b$ and $b^{2}$ is a section from the same slice. Superficial is to the left. In $b^{1}$ and $b^{2}$, Lef1 staining is not seen in the ventral telencephalon. In c, Lef1-positive cells (arrowheads) are seen in the ventricular zone but not in the mantle layer. d A schematic representation of Lef1 expression in slices hosting one EmT explant from a tau-GFP donor is shown in green. In
9/10 of slices analysed, Lef1 was not detected in the ventral telencephalon. However, in the remaining slice Lef1 staining was seen in the ventricular zone of the ventral telencephalon, but not in the mantle layer. e, f Sections from cultured slices hosting one tau-GFPpositive EmT explant (green) on the left half of the brain. $\mathrm{e}^{1}$ and $\mathrm{e}^{2}$ are serial sections of e. Lef1 staining is not detected in these sections. In $\mathrm{e}^{2}$, very little of the explant remains. In $\mathrm{f}$, Lef1 staining is seen in the ventricular zone around the explant (arrowheads). The dashed curves delineate the limits between the ventral telencephalon and the thalamus and/or dorsal telencephalon. The arrows in $\mathrm{b}^{2}, \mathrm{c}$ and $\mathrm{e}^{2}$ mark the $\mathrm{CP}$, which shows intense yellow non-specific staining. Abbreviations: $C P$ choroid plexus, EmT Eminentia thalami, $d m t$ dorsomedial telencephalon, nctx neocortex, PTh prethalamus, Th thalamus, Vtel ventral telencephalon. Scale bars $\mathrm{b}=250 \mu \mathrm{m}, \mathrm{b}^{1}, \mathrm{~b}^{2},=100 \mu \mathrm{m} ; \mathrm{c}$, $\mathrm{e}^{1}, \mathrm{e}^{2}, \mathrm{f}=50 \mu \mathrm{m}$ 
the slice culture located deep within the explant. The position of the explant for those sections is shown in Fig. $7 b-b^{3}$. High power confocal images for these slice explants immunoreacted with Ascl1 and Lef1 showed that most of these cells either expressed Lef1 or Ascl1 (Fig. $7 \mathrm{e}^{1}-\mathrm{e}^{4}$ ). Further analysis of the identity of the Lef1positive cells using in situ hybridization for Foxgl revealed that the Lef1-expressing cells in the ventral telencephalon also expressed Foxgl (not shown).

We further examined whether the Lef1-positive cells induced in the ventral telencephalic tissue surrounding the explant expressed the dorsal telencephalic marker Pax6. Lef1-positive cells intermingling with Ascl1-positive cells located lateral to the explant in Fig. $7 \mathrm{f}^{1}$ were immunonegative for Pax6 (not shown). However, a group of Lef1-positive cells located dorsal to this explant (Fig. $7 \mathrm{f}^{1}, \mathrm{f}^{2}$ ), in a region that does not normally express Pax6, were also positive for Pax6 (Fig. $7 \mathrm{f}^{3}, \mathrm{f}^{4}$ ). Interestingly, at this location which would not normally express Ascl1 either, no Ascl1-positive cells were observed (not shown). This suggests that the molecular fates of cells affected by the explant might depend on their location and pre-existing molecular state.

All together, the above results indicate that EmT tissue has the capacity to activate $\mathrm{Wnt} / \beta$-catenin signalling in the ventral telencephalon, a brain region where it is not normally active, and to suppress expression of the ventral telencephalic marker Ascl1. Abnormal expression of Pax6 in the ventral telencephalon suggests that the presence of EmT may, in certain circumstances, cause the ventral telencephalon to adopt a dorsal fate.

\section{Discussion}

The EmT is a diencephalic structure that lies between the choroid plexus of the lateral ventricle and the prethalamus (Puelles and Rubenstein 2003 and Fig. 1). Although its location has been carefully mapped in vertebrates, including humans, using anatomical landmarks and gene expression patterns (Abellan et al. 2010; Fotaki et al. 2006, 2008; Puelles et al. 2000; Retaux et al. 1999; Roy et al. 2014), little is known about its function. In contrast to nonmammalian EmT, which has been identified both during development and in adults (Wullimann and Mueller 2004), the mammalian EmT seems to be a transient developmental structure (Keyser 1972). Based on its transient appearance, characterized by strong calretinin immunoreactivity which becomes undetectable after E17.5, Abbott and Jacobowitz (1999) suggested that the EmT may act as diencephalic organizing centre. A recent study suggested that the cortical hem, ventral telencephalic septum, and EmT may act as a multicomponent patterning and organizing centre for the medial forebrain (Roy et al. 2014). Here, we examine the molecular and cellular properties of the EmT and provide strong evidence that is in line with the notion that this transient developmental mammalian structure may act as a signalling centre of the developing forebrain.

Our detailed analysis of expression of members of the Wnt, Bmp and Fgf gene families in the developing EmT revealed that, indeed, this structure is rich in expression of signalling molecules. For most of these molecules, expression was detected in the proliferative layer of the EmT at around E11.5 and ceased by E15.5, when most of the progenitor cells have differentiated into neurons, as reflected by the thinning of the size of the ventricular zone and the thickening of Calretinin staining. Unlike the neighbouring cortical hem, a secondary organizer of the hippocampal primordium (Mangale et al. 2008), which displays strong expression for both Wnt and Bmp molecules (Grove et al. 1998), the EmT showed strong expression for Wnts but low levels of expression for members of the Bmp family. Fgfs showed restricted expression in the EmT but expression of $M k p 3$, a downstream negative regulator of Fgf signalling ( $\mathrm{Li}$ et al. 2007), was more widespread. This compares to the restricted expression of $F g f 8$ in relation to the widespread expression domain of $M k p 3$ in the isthmus (Echevarria et al. 2005), a secondary organizer of the midbrain-hindbrain region (Martinez 2001).

In addition to strong expression of Wnt molecules, mainly $W n t 7 b$ and $W n t 8 b$, the EmT showed expression of downstream targets of the $\mathrm{Wnt} / \beta$-catenin pathway such as Axin2 and Lef1. Axin2, although strong, was only found in the most lateral aspect of the EmT in its entire rostrocaudal axis. This restricted expression may be due to the strong expression of Sfrp2, an antagonist of Wnt signalling (Wawrzak et al. 2007), expressed in a high-medial to lowlateral gradient complementary to that of Axin2. Very low Lefl expression in the EmT with high levels in the neighbouring prethalamus resembles lack of Lefl expression in the cortical hem with high expression levels in the adjacent dorsomedial pallium (Fotaki et al. 2011; Galceran et al. 2000).

Can the secreted proteins found to be expressed in the EmT elicit a signalling response? The fact that our heterotopic explants of EmT donor tissue within the ventral telencephalic host led to upregulation of Lef1 in the surrounding ventral telencephalon in $9 / 10$ of slices examined favours our hypothesis that signals emitted from the EmT may affect the fate of surrounding cells. Although very faintly Lef1-expressing cells were detected in the VZ of the ventral telencephalon in wild type embryos, none were found in the mantle layer. In contrast, the Lef1-expressing cells around the explant were strongly expressing cells and in $7 / 10$ cases they were not restricted to the VZ, but were also found in the mantle layer, strongly indicating that the 
grafted EmT induces production of these cells. The use of tau-GFP mice that express ubiquitously GFP (Pratt et al. 2000) allowed us to unequivocally distinguish donor from host tissue. Although the EmT has been previously shown to be a source of neurons that migrate to the ventral forebrain (Tissir et al. 2009), in our case the Lef1-positive cells were GFP-negative, indicating that it is unlikely that these cells are EmT-migrating cells. As we only observed Lef1 upregulation in the VZ in $1 / 10$ of control cases that involved transplanted cortical tissue in the ventral telencephalon, we are confident that our results are specific to EmT activity on the host environment rather than the outcome of a stochastic event. It is noteworthy that the effect of the EmT on the host tissue was only observed when two EmT pieces were grafted. It may be that although the EmT possesses an apparent ability to induce Lef1 in the ventral telencephalon ex vivo, under our experimental conditions the level of signal(s) secreted by one piece of EmT tissue does not reach the required threshold for this induction to take place.

It is also possible that the effect exerted on the surrounding tissue depends on the position of the explant. In the current experimental design, the explants were placed away from the VZ but placing them nearer to it might have led the EmT donor-emitted Wnt signals to cause a more profound effect on the surrounding tissue than the one we have currently observed. This is in line with the fact that in our CHIR-induced cultures we observed a clearer upregulation of Lef1-positive cells in the VZ rather than the mantle region.

Based on the fact that among the signalling factors we studied, Wnt $7 b$ and Wnt $8 b$ displayed the most robust and prolonged expression, we hypothesized that the primary pathway activated by the EmT is the $\mathrm{Wnt} / \beta$-catenin signalling cascade. In cultured ventral telencephalic slices, where $\mathrm{Wnt} / \beta$-catenin signalling was activated by inhibition of Gsk $3 \beta$ activity (van Noort et al. 2002), Lef1 expression increased with an increased inhibition of this activity. The high levels of Lef1 expression in DMSO-treated slices have been previously observed in other culture systems suggesting that DMSO per se may activate Wnt/ $\beta$-catenin signalling (Nakamura et al. 2003; Peng et al. 2002). However, these were significantly lower than the number of Lef1-expressing cells observed with even the lowest concentration of drug activator, confirming that the significant increase in Lef1-expressing cells in the ventral telencephalon was the result of a specific activation of the $\mathrm{Wnt} / \beta$-catenin pathway. Although some of the Lef1-positive cells co-expressed Ascl1, the number of these Lef1;Ascl1-positive cells was very small and remained unchanged among all treated conditions and the control group, suggesting that these double-labelled cells may
Fig. 6 Lef1 expression in the ventral telencephalon of cultured slices hosting two EmT explants. a A schematic representation of Lef1 expression in this group. The region of the tau-GFP donor tissue from which the two EmTs were taken from is shown in green. In 1/10 of slices analysed, Lef1 was not detected in the ventral telencephalon; in 2/10 slices, Lef1 was seen only in the ventricular zone (VZ); and in $7 / 10$ slices, Lef1 was seen in the VZ and mantle layer. (b) Double immunofluorescence for GFP (green) and Calretinin (CR) (red) on a section from a cultured slice hosting two EmT explants (asterisk) on the left half of the brain. $\mathbf{b}^{\mathbf{1}}$ is a magnification of the region indicated in $b$. The explants are both GFP and Calretinin positive confirming that these are EmT tissue. c Lef1 immunohistochemistry (brown staining) counterstained with cresyl violet (blue staining) on a consecutive section to that shown in $\mathrm{b}$ reveals staining in cells peripheral to the explants (green arrowhead). These Lef1-positive cells surrounding the explants are not GFP-positive as inferred from their positions in $\mathrm{b}^{1}$ (white arrowheads) and $\mathrm{c}^{1}$ (green arrowheads). $\mathbf{b}^{\mathbf{1}}$ and $\mathrm{c}^{\mathbf{1}}$ are magnification of the regions indicated in $\mathrm{b}$ and $\mathrm{c}$, respectively. $\mathbf{c}^{\mathbf{2}}$ is a magnification of the region indicated in $\mathbf{c}^{\mathbf{1}}$. $\mathbf{d}$ and e are serial sections from a slice hosting two EmT explants on one half of the ventral telencephalon. In e, where there is no EmT explant remaining, Lef1 staining is found in the $\mathrm{VZ}$ and mantle layer. The arrowhead points to Lef1 expression limited at the ventricular zone of the untreated hemisphere. $\mathbf{e}^{\mathbf{1}}$ is a magnification of the Vtel shown in e. Lef1-positive cells found in the ventral telencephalon (arrowheads) are not GFP-positive suggesting that they may be host cells. The section is counterstained with DAPI (blue). The dashed curves delineate the limits between the ventral telencephalon and the thalamus and/or dorsal telencephalon. Abbreviations: $C P$ choroid plexus; $d m t$ dorsomedial telencephalon, $E m T$ eminentia thalami; $n c t x$ neocortex; PTh prethalamus, Th thalamus, Vtel ventral telencephalon. Scale bars $\mathrm{b}, \mathrm{c}, \mathrm{d}, \mathrm{e}=250 \mu \mathrm{m} ; \mathrm{b}^{1}, \mathrm{c}^{1}, \mathrm{e}^{1}=50 \mu \mathrm{m} ; \mathrm{c}^{2}=20 \mu \mathrm{m}$

account for the low number of Lef1-positive cells normally found in the ventral telencephalon.

The dose-dependent induction of Lef1 in these ventral slices was concomitant with a significant dose-dependent decrease in Ascl1 expression, strongly suggesting that Lef1 upregulation in the ventral telencephalon leads to a suppression of ventral fates. Similarly, when the induced Lef1positive cells in our explant experiment were examined for expression of the ventral telencephalic marker Ascl1 and the telencephalic marker Foxg1, it was found that these cells were Ascl1-negative;Foxg1-positive. Thus, results of these two different experimental approaches suggest that Wnt/ $\beta$ catenin signalling may lead to a cell fate change in the surrounding tissue. It is worth noting that some of the Lef1induced cells were in deeper positions, relative to the VZ apical surface, than those that showed induction in the CHIR-treated explants. One likely explanation for this is that the EmT grafts produce signalling molecules additional to Wnts such as Fgfs and Bmps, which may affect exactly which cells undergo Lef1 activation in the host tissue.

If the EmT-derived signals inhibit Ascl1-expressing cells, why are there still a lot of Ascl1-positive cells surrounding the EmT explants? We propose that the EmTsecreted signals are able to affect the fate of some cells which respond to those signals by upregulating Lef1. One 


\section{CULTURED SLICES WITH TWO EmT EXPLANTS (GROUP 2)}
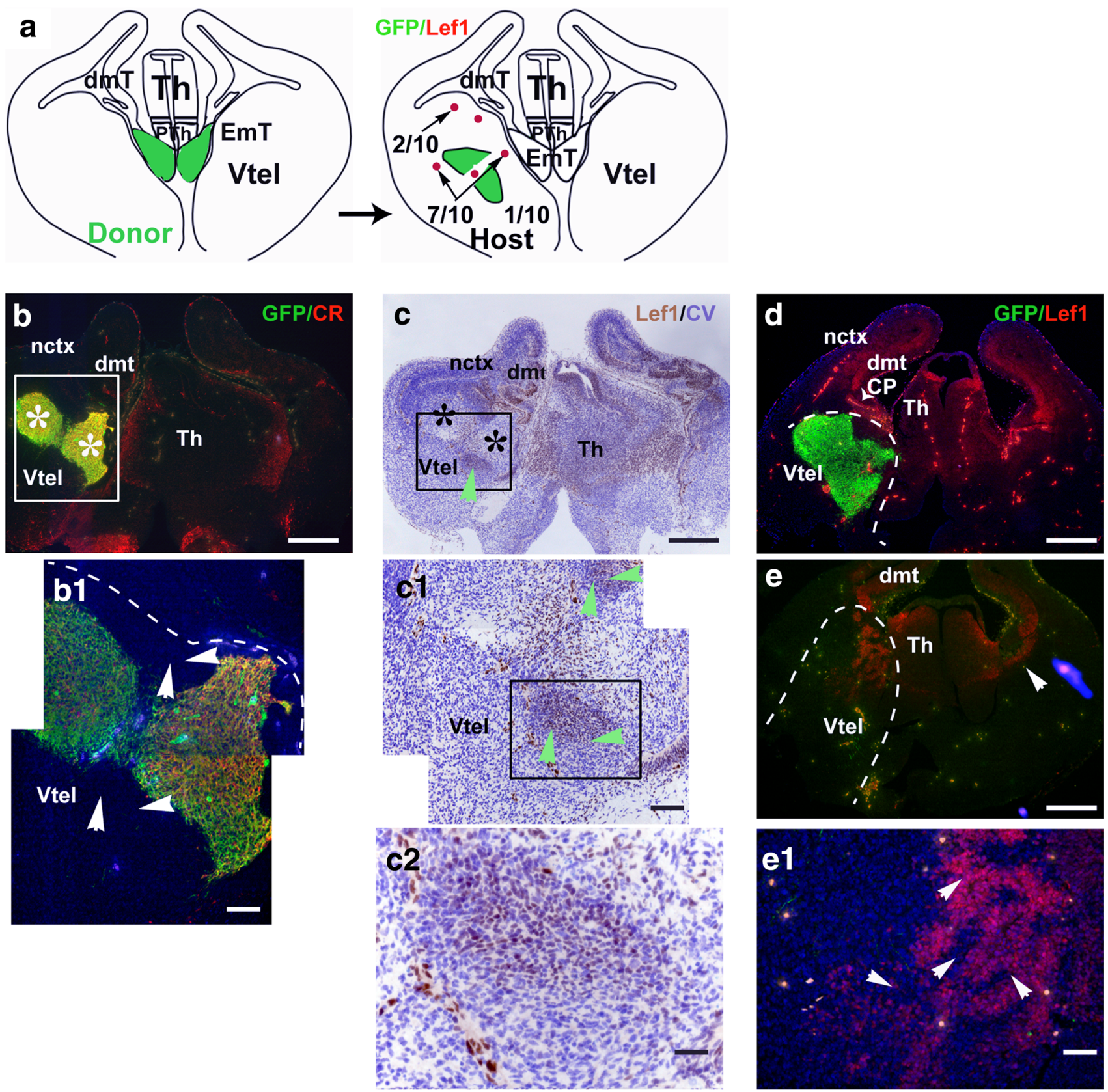

reason that some cells do not respond by upregulating Lef1 may be that their state of differentiation renders them unresponsive to the signal. Alternatively, the levels of signal reaching each cell may differ among cells due to their precise position and the nature of the diffusion of the signal from the EmT explant. In addition, as discussed above, the EmT expresses different secreted factors along its rostrocaudal and medio-lateral axis and their expression levels change during embryonic development. In line with this dynamic spatio-temporal expression, EmT cells may elicit a different response at different positions in the surrounding tissue due to spatial variation in the cocktail of molecules produced by the explants and/or in the relative amounts of these secreted factors. 
In agreement with the role of $\mathrm{Wnt} / \beta$-catenin signalling in dorsal telencephalic specification (Backman et al. 2005; Gunhaga et al. 2003) and the fact that constitutive expression of $\beta$-catenin in the mouse ventral telencephalon leads to its partial dorsalization with a concomitant repression of ventral telencephalic markers (Backman et al. 2005), it is possible that these Lef1-positive cells have acquired dorsal telencephalic identity. This is further supported by the upregulated expression of the dorsal telencephalic marker Pax6 within the ventral telencephalon adjacent to the EmT explant. It is intriguing that this Pax6 expression was observed in cells ectopically expressing Lef1 that were not surrounded by Ascl1 expressing cells but was absent from Lef1-positive cells surrounded by Ascl1-positive cells. Further studies with additional markers will be needed to test further the identities of the EmT-induced Lef1-positive cells within the ventral telencephalon and to allow us to understand the necessary conditions that trigger those identities.

A self-evident question that this study raises is that if the EmT plays a role as signalling centre, which are the domains it exerts its signalling activity on? Signalling centres usually lie at the borders of the regions they specify (Kiecker and Lumsden 2005). As the EmT lies between the choroid plexus of the lateral ventricle, the ventral telencephalon and the prethalamus, an attractive hypothesis is that it may pattern these regions (Online Resources 1, 2 $\& 3)$. The phenotypic defects observed in mouse mutants with abnormal EmT structures favour this hypothesis. In the Gli3 mutant, EmT cell clusters intermingle with dorsal telencephalic cells possibly as a result of abnormal formation of the telencephalic-diencephalic boundary (Fotaki et al. 2006). In addition, in Gli3 chimaeras the EmT seems to become duplicated near the telencephalic-diencephalic border, where nearby cells express abnormal sets of transcription factors-cells lying on the telencephalic side of the boundary express diencephalic markers (Quinn et al. 2009). In the Lhx2 mutant, the EmT expands at the expense of the caudal ganglionic eminence, leading to the hypothesis that the EmT may act as the diencephalic hem to regulate key aspects of forebrain patterning and development (Roy et al. 2014). In the Olig 2 mutant, the EmT is expanded at the expense of a hypoplastic prethalamus (Ono et al. 2014). In all the above examples, changes in the mutant EmT are concomitant with significant changes in the above-mentioned neighbouring forebrain tissue.

Our findings show that the EmT not only expresses Wnt molecules but also activates $\mathrm{Wnt} / \beta$-catenin signalling. At present, we can only hypothesize about the forebrain areas to which the EmT Wnts may be signalling. Based on the fact that $\beta$-catenin promotes and maintains thalamic fate but prevents prethalamic fate (Bluske et al. 2012), it is unlikely that EmT-derived Wnt/ $\beta$-catenin signals target the
Fig. 7 Ascl1, Lef1 and Pax6 expression in the ventral telencephalon of cultured slices. a A schematic representation of Ascl1 (green dots) and Lef1 (red dots) expression observed in slices with EmT explants. In sections with explants present, Ascll was not seen within the explants (depicted with a black outline) but was found in ventral telencephalic cells surrounding them. In slices hosting two EmT explants, Lef1-positive cells mingled with Ascl1-positive cells within the ventral telencephalon and outside the explant region. $\mathbf{b}-\mathbf{b}^{\mathbf{3}}$ Serial cryostat sections of a slice culture with two tau-GFP EmT explants on the left hemisphere. GFP immunofluorescence shows explant position in $\mathbf{b}$. Sections $\left(\mathbf{b}^{\mathbf{1}}-\mathbf{b}^{\mathbf{3}}\right)$ are serial sections located in deeper layers to b. In these sections, the GFP staining is gradually reduced $\left(\mathbf{b}^{\mathbf{1}}, \mathbf{b}^{\mathbf{2}}\right)$ and eventually disappears $\left(\mathbf{b}^{\mathbf{3}}\right)$. c A section with an EmT explant present (outlined in white). Ascll staining is seen in the ventricular zone and mantle layer around the explant but not in the explant per se. $\mathbf{d}, \mathbf{d}^{\mathbf{1}}$ Ascl1 and Lef1 expression in the experimental half of a slice hosting two EmT explants. Both Ascl1-positive (green) and Lef1-positive (red) cells are seen in the ventricular zone and mantle layer of the ventral telencephalon. The dotted square in d outlines the high power image in $d^{1}$. $d$ is a section adjacent to $b^{3}$. As with $b^{3}$, the explant is not visible anymore in $\mathrm{d}$. However, its position corresponds to that of the dotted squares in panels $\mathrm{b}-\mathrm{b}^{3}$. At higher magnifications $\left(\mathbf{e}^{\mathbf{1}}-\mathbf{e}^{\mathbf{4}}\right)$, many single-labelled Lef1-positive cells (arrows in $\mathrm{e}^{3}$ ) are found mingling with single-labelled Ascl1-expressing cells (arrows in $\mathrm{e}^{2}$ ). Blue staining $\left(\mathrm{b}-\mathrm{b}^{3} ; \mathrm{e}^{1}-\mathrm{e}^{4}\right)$ is DAPI counterstain. $\mathbf{f}^{\mathbf{1}}-\mathbf{f}^{\mathbf{4}}$ Lef1 and Pax6 expression in the experimental half of a host slice. The blue staining in $\mathbf{f}^{\mathbf{1}}$ corresponds to the tau-GFP EmT explant. Dorsal to the explant, a group of Lef1-positive cells are found ( $r e d$ in $\mathrm{f}^{2}$ ). Most of these cells seem to co-express the dorsal telencephalic marker Pax6 (green in $\mathbf{f}^{\mathbf{3}}$, $\mathbf{f}^{\mathbf{4}}$ ). The dotted square in $\mathrm{f}^{1}$ corresponds to the high magnifications in $\mathrm{f}^{2}-\mathrm{f}^{3}$. The arrows in $\mathrm{f}^{3}$ point to Pax6-positive cells within the GFPpositive EmT. Abbreviations: dmt dorsomedial telencephalon; EmT eminentia thalami; nctx neocortex; PTh prethalamus, Th thalamus, Vtel ventral telencephalon. Scale bars $\mathrm{b}_{-} \mathrm{b}^{3}, \mathrm{f}^{1}=200 \mu \mathrm{m} ; \mathrm{c}$, $\mathrm{d}=100 \mu \mathrm{m} ; \mathrm{d}^{1}, \mathrm{f}^{2}-\mathrm{f}^{4}=50 \mu \mathrm{m} ; \mathrm{e}^{1}-\mathrm{e}^{4}=25 \mu \mathrm{m}$

neighbouring prethalamus. An attractive hypothesis is that, similar to the Wnt-rich cortical hem which has been proposed to affect choroid plexus development (Grove et al. 1998), Wnt signals from the EmT also affect formation and/or function of the adjacent choroid plexus. This is in line with the fact that already at 8 somites, before choroid plexus formation, a $W n t 7 b / W n t 8 b$ rich domain defines a region of the prosencephalon that probably corresponds to the future EmT and adjacent cortical hem (Fotaki et al. 2011).

A comprehensive fate map of the mammalian EmT has not been reported to date. It has been shown that pioneer neurons from the mouse EmT migrate to the ganglionic eminences and the marginal zone of the cortex (MoranteOria et al. 2003) and also form part of the ventral forebrain (Tissir et al. 2009). It has also been reported that the EmT gives rise to the bed nucleus of the stria medullaris and that of the posterior part of the stria terminalis (Puelles et al. 2000). Although our current study cannot provide any information on the fate of the EmT, adapting our experimental protocol using homotopic explants from tau-GFP mouse donors could generate valuable data regarding the fate of EmT cells. 

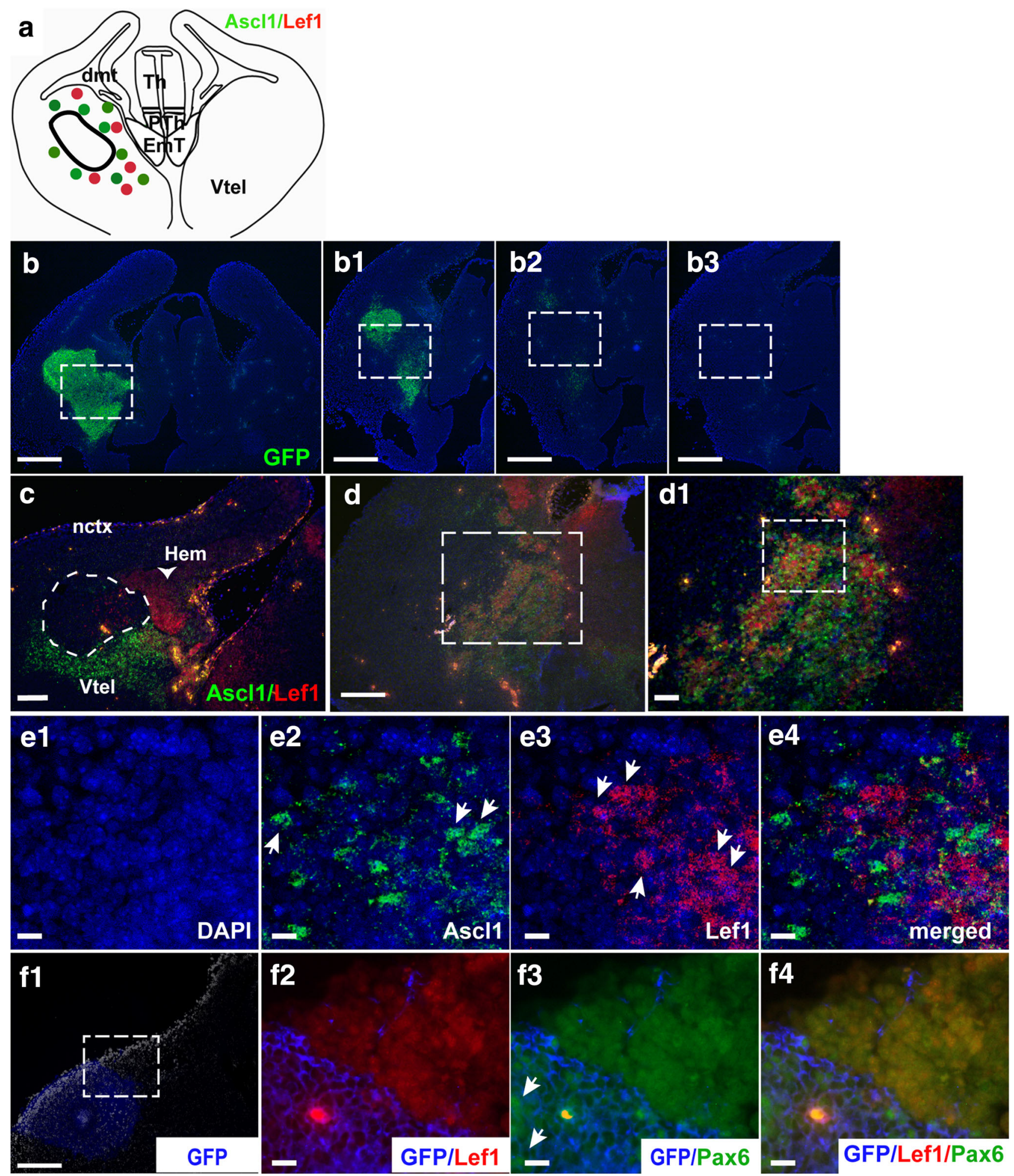

\section{Conclusions}

The EmT is a Wnt-rich expressing region with an ability to induce the fate of surrounding cells away from its original source. These properties strongly support its role as a forebrain signalling centre. Future experiments will explore further this potential and will also examine the properties of the other signalling molecules expressed in the EmT. 
Acknowledgments This work was supported by the Medical Research Council (MR/J013137/1 to VF, MR/G0800429 to DJP and MR/G0801359 to TT). KKAO was supported by an Overseas Research Studentship (ORS) award and a fellowship from the University of Ghana. The sponsors had no involvement in the design of the study or the writing of this manuscript. We thank Rowena Smith, Mike Molinek and Dimitris Amanitis for assistance with the in situ hybridizations, Trudi Guillespi and the IMPACT facility for the confocal imaging and staff from the CBS-HRB animal facility for mouse husbandry. We thank Thomas Pratt for providing the TgTP6.3 mice and Nuria Ruiz Reig for useful discussions. We also thank the following researchers for providing plasmids for riboprobe synthesis and antibodies: J Galceran (Lef1), BG Hermmann (Mkp3), R Hevner (Tbr2), R Hindges (Bmp4), O Machon (Axin2), P Murphy (Wnt9a), A Rattner (Sfrp2), J Rubenstein (Fgf8), S Vainio (Spry 1), T Yu (Fgf15). The Pax6 monoclonal antibody was developed by A Kawakami and the Lim $1+2$ by TM Jessell and S Brenner-Morton and were obtained from the Developmental Studies Hybridoma Bank, created by the NICHD of the NIH and maintained at The University of Iowa, Department of Biology, Iowa City, IA 52242.

\section{Compliance with ethical standards}

Conflict of interest The authors declare that they have no conflict of interest.

Open Access This article is distributed under the terms of the Creative Commons Attribution 4.0 International License (http://crea tivecommons.org/licenses/by/4.0/), which permits unrestricted use, distribution, and reproduction in any medium, provided you give appropriate credit to the original author(s) and the source, provide a link to the Creative Commons license, and indicate if changes were made.

\section{References}

Abbott LC, Jacobowitz DM (1999) Developmental expression of calretinin-immunoreactivity in the thalamic eminence of the fetal mouse. Int J Dev Neurosci 17:331-345

Abellan A, Vernier B, Retaux S, Medina L (2010) Similarities and differences in the forebrain expression of Lhx 1 and Lhx5 between chicken and mouse: insights for understanding telencephalic development and evolution. J Comp Neurol 518:3512-3528. doi: $10.1002 / \mathrm{cne} .22410$

Backman M, Machon O, Mygland L, van den Bout CJ, Zhong W, Taketo MM, Krauss S (2005) Effects of canonical Wnt signaling on dorso-ventral specification of the mouse telencephalon. Dev Biol 279:155-168. doi:10.1016/j.ydbio.2004.12.010

Bluske KK, Vue TY, Kawakami Y, Taketo MM, Yoshikawa K, Johnson JE, Nakagawa Y (2012) $\beta$-Catenin signaling specifies progenitor cell identity in parallel with Shh signaling in the developing mammalian thalamus. Development 139:2692-2702. doi:10.1242/dev.072314

Echevarria D, Martinez S, Marques S, Lucas-Teixeira V, Belo JA (2005) Mkp3 is a negative feedback modulator of Fgf8 signaling in the mammalian isthmic organizer. Dev Biol 277:114-128. doi:10.1016/j.ydbio.2004.09.011

Flames N, Pla R, Gelman DM, Rubenstein JL, Puelles L, Marin O (2007) Delineation of multiple subpallial progenitor domains by the combinatorial expression of transcriptional codes. J Neurosci 27:9682-9695. doi:10.1523/JNEUROSCI.2750-07.2007

Fotaki V, Yu T, Zaki PA, Mason JO, Price DJ (2006) Abnormal positioning of diencephalic cell types in neocortical tissues in the dorsal telencephalon of mice lacking functional Gli3. J Neurosci 26:9282-9292

Fotaki V, Price DJ, Mason JO (2008) Newly identified patterns of Pax2 expression in the developing mouse forebrain. BMC Dev Biol 8:79. doi:10.1186/1471-213X-8-79

Fotaki V, Price DJ, Mason JO (2011) Wnt/beta-catenin signaling is disrupted in the extra-toes (Gli3(Xt/Xt)) mutant from early stages of forebrain development, concomitant with anterior neural plate patterning defects. J Comp Neurol 519:1640-1657. doi:10.1002/cne.22592

Fotaki V, Smith R, Pratt T, Price DJ (2013) Foxg1 is required to limit the formation of ciliary margin tissue and Wnt/beta-catenin signalling in the developing nasal retina of the mouse. Dev Biol 380:299-313. doi:10.1016/j.ydbio.2013.04.017

Galceran J, Miyashita-Lin EM, Devaney E, Rubenstein JL, Grosschedl R (2000) Hippocampus development and generation of dentate gyrus granule cells is regulated by LEF1. Development 127:469-482

Grove EA, Fukuchi-Shimogori T (2003) Generating the cerebral cortex area map. Annu Rev Neurosci 26:355-383

Grove EA, Tole S, Limon J, Yip L-W, Ragsdale CW (1998) The hem of the embryonic cerebral cortex is defined by thr expression of multiple Wnt genes and is compromised in Gli3-deficient mice. Development 125:2315-2325

Guillemot F, Joyner AL (1993) Dynamic expression of the murine achaete-scute homologue mash-1 in the developing nervous system. Mech Dev 42:171-185

Gunhaga L, Marklund M, Sjodal M, Hsieh JC, Jessell TM, Edlund T (2003) Specification of dorsal telencephalic character by sequential Wnt and FGF signaling. Nat Neurosci 6:701-707. doi: $10.1038 / \mathrm{nn} 1068$

Hanashima C, Shen LJ, Li SC, Lai E (2002) Brain factor-1 controls the proliferation and differentiation of neocortical progenitor cells through independent mechanisms. J Neurosci 22:6526-6536

Houart C, Westerfield M, Wilson SW (1998) A small population of anterior cells pattern the forebrain during zebrafish gastrulation. Nature 391:788-792

Houart C, Caneparo L, Heisenberg C-P, Barth AK, Masaya T-U, Wilson SW (2002) Establishment of the Telencephalon during gastrulation by local antagonim of Wnt signaling. Neuron 35:255-265

Hsu SC, Galceran J, Grosschedl R (1998) Modulation of transcriptional regulation by LEF-1 in response to Wnt- 1 signaling and association with beta-catenin. Mol Cell Biol 18:4807-4818

Jho EH, Zhang T, Domon C, Joo CK, Freund JN, Costantini F (2002) $\mathrm{Wnt} /$ beta-catenin/Tcf signaling induces the transcription of Axin2, a negative regulator of the signaling pathway. Mol Cell Biol 22:1172-1183

Keyser A (1972) The development of the diencephalon of the Chinese hamster. An investigation of the validity of the criteria of subdivision of the brain. Acta Anat Suppl 59:1-178

Kiecker C, Lumsden A (2004) Hedgehog signaling from the ZLI regulates diencephalic regional identity. Nat Neurosci 7:1242-1249. doi: $10.1038 / \mathrm{nn} 1338$

Kiecker C, Lumsden A (2005) Compartments and their boundaries in vertebrate brain development. Nat Rev Neurosci 6:553-564. doi: $10.1038 / \mathrm{Nrn} 1702$

Lee SM, Tole S, Grove E, McMahon AP (2000) A local Wnt-3a signal is required for development of the mammalian hippocampus. Development 127(3):457-467

Li C, Scott DA, Hatch E, Tian X, Mansour SL (2007) Dusp6 (Mkp3) is a negative feedback regulator of FGF-stimulated ERK signaling during mouse development. Development 134:167-176. doi:10.1242/dev.02701 
Liu A, Joyner AL (2001) Early anterior/posterior patterning of the midbrain and cerebellum. Annu Rev Neurosci 24:869-896. doi:10.1146/annurev.neuro.24.1.869

Lo LC, Johnson JE, Wuenschell CW, Saito T, Anderson DJ (1991) Mammalian acaete-scute homolog 1 is transiently expressed by spatially restricted subsets of early neuroepithelial and neural crest cells. Genes Dev 5:1524-1537

Magnani D, Hasenpusch-Theil K, Jacobs EC, Campagnoni AT, Price DJ, Theil T (2010) The Gli3 hypomorphic mutation Pdn causes selective impairment in the growth, patterning, and axon guidance capability of the lateral ganglionic eminence. J Neurosci 30:13883-13894. doi:10.1523/JNEUROSCI.3650-10.2010

Mangale VS et al (2008) Lhx2 selector activity specifies cortical identity and supresses hippocampal organizer fate. Science 319:304-309

Maretto S et al (2003) Mapping Wnt/beta-catenin signaling during mouse development and in colorectal tumors. Proc Nat Acad Sci 100:3299-3304. doi:10.1073/pnas.0434590100

Martinez S (2001) The isthmic organizer and brain regionalization. Int J Dev Biol 45:367-371

Martinez S, Alvarado-Mallart R-M (1989) Rostral cerebellum originates from the caudal portion of the so-called 'mesencephalic' vesicle: a study using chick/quail chimeras. Eur J Neurosci 1:549-560. doi:10.1111/j.1460-9568.1989.tb00362.x

Martinez S, Wassef M, Alvarado-Mallart R-M (1991) Induction of a mesencephalic phenotype in the 2-Day-Old chick prosencephalon is preceded by the early expression of the homeobox gene en. Neuron 6:971-981. doi:10.1016/08966273(91)90237-T

Martinez-Ferre A, Martinez S (2012) Molecular regionalization of the diencephalon. Front Neurosci 6:73. doi:10.3389/fnins.2012. 00073

Mason I (2007) Initiation to end point: the multiple roles of fibroblast growth factors in neural development. Nat Rev Neurosci 8:583-596. doi:10.1038/nrn2189

Meyer G (2010) Building a human cortex: the evolutionary differentiation of Cajal-Retzius cells and the cortical hem. J Anat 217:334-343. doi:10.1111/j.1469-7580.2010.01266.x

Morante-Oria J, Carleton A, Ortino B, Kremer EJ, Fairén A, Lledo PM (2003) Subpallial origin of a population of projecting pioneer neurons during corticogenesis. Proc Natl Acad Sci 100:12468-12473

Nakamura T, Sano M, Songyang Z, Schneider MD (2003) A Wntand beta -catenin-dependent pathway for mammalian cardiac myogenesis. Proc Natl Acad Sci 100:5834-5839. doi:10.1073/ pnas.0935626100

Ono K et al (2014) Development of the prethalamus is crucial for thalamocortical projection formation and is regulated by Olig2. Development 141:2075-2084. doi:10.1242/dev.097790

Peng CF, Wei Y, Levsky JM, McDonald TV, Childs G, Kitsis RN (2002) Microarray analysis of global changes in gene expression during cardiac myocyte differentiation. Physiol Genom 9:145-155. doi:10.1152/physiolgenomics.00027.2002

Porfiri E, Rubinfeld B, Albert I, Hovanes K, Waterman M, Polakis P (1997) Induction of a beta-catenin-LEF-1 complex by wnt-1 and transforming mutants of beta-catenin. Oncogene 15:2833-2839

Porteus MH, Bulfone A, Liu JK, Puelles L, Lo LC, Rubenstein JLR (1994) DLX-2, MASH-1 and MAP-2 expression and bromodeoxyuridine incorporation define molecularly distinct cell populations in the embyonic mouse forebrain. $\mathbf{J}$ Neurosci 14:6370-6383

Pratt T, Sharp L, Nichols J, Price DJ, Mason JO (2000) Embryonic stem cells and transgenic mice ubiquitously expressing a tautagged green fluorescent protein. Dev Biol 228:19-28. doi:10. 1006/dbio.2000.9935
Puelles L, Rubenstein JL (2003) Forebrain gene expression domains and the evolving prosomeric model. Trends Neurosci 26:469-476. doi:10.1016/S0166-2236(03)00234-0

Puelles L et al (2000) Pallial and subpallial derivatives in the embryonic chick and mouse telencephalon, traced by the expression of genes Dlx-2, Emx 1, Pax-6, and Tbr-1. J Comp Neurol 424:409-438

Quinn JC, Molinek M, Mason JO, Price DJ (2009) Gli3 is required autonomously for dorsal telencephalic cells to adopt appropriate fates during embryonic forebrain development. Dev Biol 327:204-215. doi:10.1016/j.ydbio.2008.12.008

Raible F, Brand M (2004) Divide et Impera-the midbrain-hindbrain boundary and its organizer. Trends Neurosci 27:727-734. doi:10. 1016/j.tins.2004.10.003

Retaux S, Rogard M, Bach I, Failli V, Besson M-J (1999) Lhx-9: a novel LIM-homeodomain gene expressed in the forebrain. J Neurosci 19:783-793

Ring DB et al (2003) Selective glycogen synthase kinase 3 inhibitors potentiate insulin activation of glucose transport and utilization in vitro and in vivo. Diabetes 52:588-595

Roy A, Gonzalez-Gomez M, Pierani A, Meyer G, Tole S (2014) Lhx2 regulates the development of the forebrain hem system. Cereb Cortex 24:1361-1372. doi:10.1093/cercor/bhs421

Shimamura K, Rubenstein JLR (1997) Inductive interactions direct regionization of the mouse forebrain. Development 124:2709-2718

Suzuki-Hirano A, Harada H, Sato T, Nakamura H (2010) Activation of Ras-ERK pathway by Fgf8 and its downregulation by Sprouty2 for the isthmus organizing activity. Dev Biol 337:284-293. doi:10.1016/j.ydbio.2009.10.044

Tao W, Lai E (1992) Telencephalo-restricted expression of BF-1, a new member of the HNF-3/fork head gene family, in the developing rat brain. Neuron 8:957-966

Theil T, Alvarez-Bolado G, Walter A, Ruther U (1999) Gli3 is required for Emx gene expression during dorsal telencephalon development. Development 126:3561-3571

Tissir F, Ravni A, Achouri Y, Riethmacher D, Meyer G, Goffinet AM (2009) DeltaNp73 regulates neuronal survival in vivo. Proc Natl Acad Sci 106:16871-16876. doi:10.1073/pnas.0903191106

Tole S, Ragsdale CW, Grove EA (2000) Dorsoventral patterning of the telencephalon is disrupted in the mouse mutant extra-toes $(\mathrm{J})$. Dev Biol 217:254-265. doi:10.1006/dbio.1999.9509

Trujillo CM, Alonso A, Delgado AC, Damas C (2005) The rostral and caudal boundaries of the diencephalon. Brain Res Brain Res Rev 49:202-210. doi:10.1016/j.brainresrev.2005.01.002

van Noort M, Meeldijk J, van der Zee R, Destree O, Clevers H (2002) Wnt signaling controls the phosphorylation status of betacatenin. J Biol Chem 277:17901-17905. doi:10.1074/jbc. M111635200

Vieira C, Garda AL, Shimamura K, Martinez S (2005) Thalamic development induced by Shh in the chick embryo. Dev Biol 284:351-363. doi:10.1016/j.ydbio.2005.05.031

Wawrzak D, Metioui M, Willems E, Hendrickx M, de Genst E, Leyns L (2007) Wnt3a binds to several sFRPs in the nanomolar range. Biochem Biophys Res Commun 357:1119-1123. doi:10.1016/j. bbrc.2007.04.069

Wolpert L (1996) One hundred years of positional information. Trends Genet 12:359-364

Wolpert L (2011) Positional information and patterning revisited. J Theor Biol 269:359-365. doi:10.1016/j.jtbi.2010.10.034

Wullimann MF, Mueller T (2004) Identification and morphogenesis of the eminentia thalami in the zebrafish. J Comp Neurol 471:37-48. doi:10.1002/cne.20011

Xuan S, Baptista CA, Balas G, Tao W, Suares VC, Lai E (1995) Winged helix factor BF-1 is essential for the development of the cerebral hemispheres. Neuron 14:1141-1152 